\title{
Non-coherent energetic interfaces accounting for degradation
}

\author{
Ali Esmaeili ${ }^{1}$ - Paul Steinmann ${ }^{1}$ - Ali Javili ${ }^{2}$
}

Received: 6 May 2016 / Accepted: 7 October 2016 / Published online: 21 November 2016

(C) Springer-Verlag Berlin Heidelberg 2016

\begin{abstract}
Within the continuum mechanics framework, there are two main approaches to model interfaces: classical cohesive zone modeling (CZM) and interface elasticity theory. The classical CZM deals with geometrically noncoherent interfaces for which the constitutive relation is expressed in terms of traction-separation laws. However, CZM lacks any response related to the stretch of the midplane of the interface. This issue becomes problematic particularly at small scales with increasing interface area to bulk volume ratios, where interface elasticity is no longer negligible. The interface elasticity theory, in contrast to CZM, deals with coherent interfaces that are endowed with their own energetic structures, and thus is capable of capturing elastic resistance to tangential stretch. Nonetheless, the interface elasticity theory suffers from the lack of inelastic material response, regardless of the strain level. The objective of this contribution therefore is to introduce a generalized mechanical interface model that couples both the elastic response along the interface and the cohesive response across the interface whereby interface degradation is taken into account. The material degradation of the interface mid-plane
\end{abstract}

Dedicated to the memory of Prof. Gérard A. Maugin (December 2, 1944 - September 22, 2016).

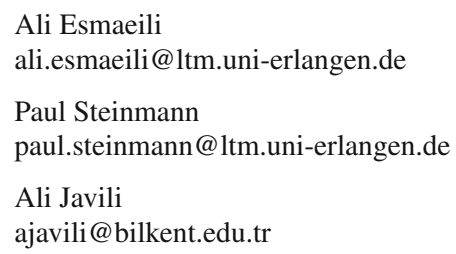

Ali Esmaeili

ali.esmaeili@1tm.uni-erlangen.de

Paul Steinmann

paul.steinmann@1tm.uni-erlangen.de

Ali Javili

ajavili@bilkent.edu.tr

1 Chair of Applied Mechanics, University of Erlangen-Nuremberg, Egerlandstrasse 5, 91058 Erlangen, Germany

2 Department of Mechanical Engineering, Bilkent University, 06800 Ankara, Turkey is captured by a non-local damage model of integral-type. The out-of-plane decohesion is described by a classical cohesive zone model. These models are then coupled through their corresponding damage variables. The non-linear governing equations and the weak forms thereof are derived. The numerical implementation is carried out using the finite element method and consistent tangents are derived. Finally, a series of numerical examples is studied to provide further insight into the problem and to carefully elucidate key features of the proposed theory.

Keywords Interface elasticity · Non-local damage · Cohesive zone $\cdot$ Nano-materials $\cdot$ Size effect $\cdot$ Generalized interfaces

\section{Introduction}

An interface ${ }^{1}$ can markedly differ from its surrounding bulk due to processes such as aging or atomic rearrangement that can substantially affect the overall response of the body. It is important to mention that such an interface is modeled as a zero-thickness entity to capture the finite thickness interphase behavior and is thus corresponding to the overall behavior of the interphase (see Fig. 1b). In other words, an interface is a lower-dimensional deformable surface embedded in three-dimensional Euclidean space representing a finite-thickness yet thin layer of material. In fact it can be shown that an elastic interface model can be captured by an asymptotically zero-thickness bulk [17]. Moreover, it is also possible to obtain the elastic modulus of interfaces by experimental tests. See for instance $[13,33]$. In addition

\footnotetext{
${ }_{1}$ An interface can be regarded as a two-sided surface, therefore the terms "surface" and "interface" are sometimes used interchangeably.
} 

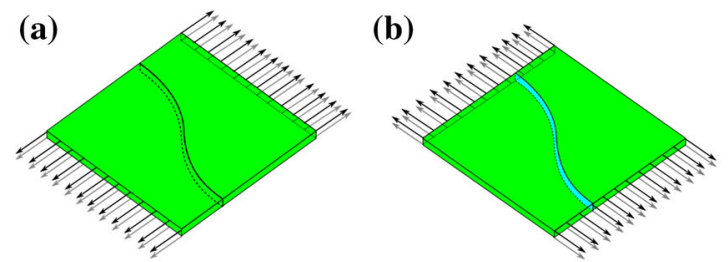

(c)

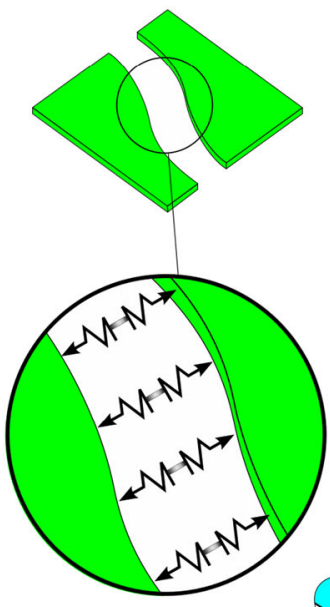

damage type

cohesive interface model $\llbracket \varphi \rrbracket \neq 0, \llbracket P \rrbracket \cdot \bar{N}=\mathbf{0}$

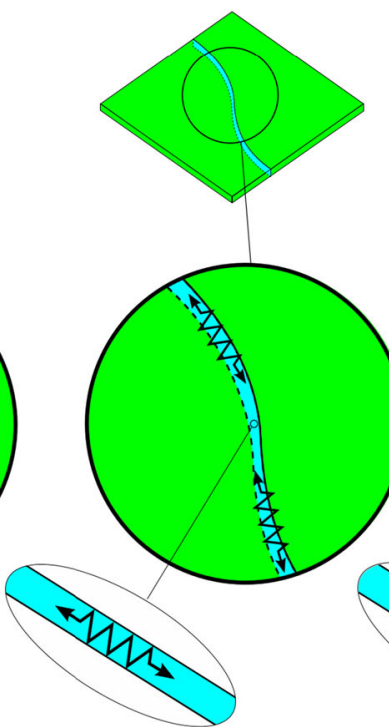
elasticity theory $\llbracket \varphi \rrbracket=0, \llbracket P \rrbracket \cdot \bar{N} \neq 0$

Fig. 1 a A loading "normal" to the interface causing opening across the interface modeled by the cohesive zone approach; $\mathbf{b}$ a loading "parallel" to the interface causing no opening across the interface yet deforming the interface in its tangential plane, neglecting interface damage, modeled by interface elasticity theory; c a loading "parallel" to the interface causing no opening across the interface yet deforming the interface in its tangential plane, accounting for interface damage, modeled by interface

to experimental methods, the surface elastic properties are obtainable utilizing semi-analytic methods, ab-initio calculations, atomistic simulation, surface Cauchy-Born techniques or phase-field approaches [see [21,23,54,71,94], respectively, for further details]. The classical interface/surface elasticity theory proposed by $[38,61]$ deals with modeling the above described interfaces. Within this theory the interface is assumed to be coherent, ${ }^{2}$ is endowed with its own elastic behavior or more precisely with its own energetic structures and proves to be a very powerful tool to capture the material behavior at the nanometer scale where the surface/interface area to the bulk volume ratio is significant. Due to the above features, these interfaces are labeled elastic or energetic. For further details see for instance, $[12,20,22,29,30,36,39,43-46,48-50,54,58,82,84]$ and ref-

${ }^{2}$ Recall that the coherence condition on the interface implies the continuity of the displacement across the interface and thus the displacement jump vanishes identically.

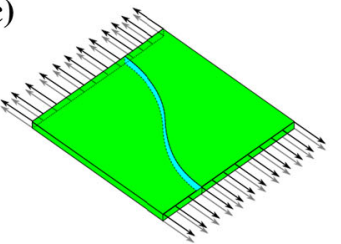

(d)
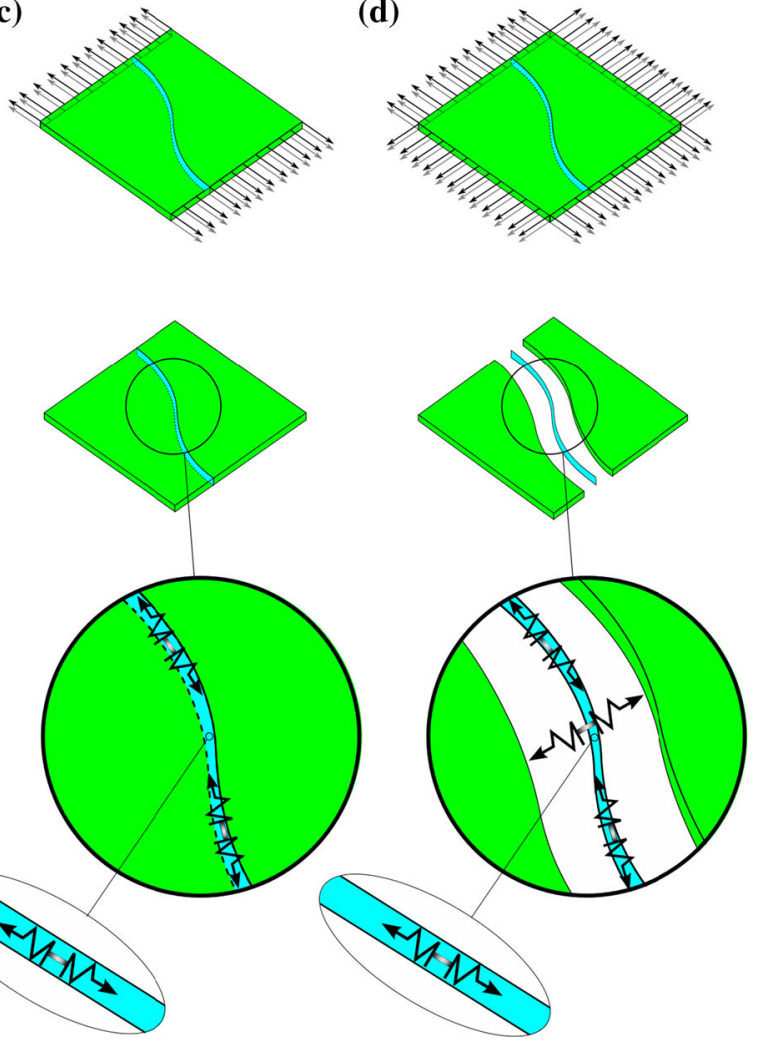
interface elasticity accounting for damage

interface inelasticity + $\llbracket \varphi \rrbracket=0, \llbracket P \rrbracket \cdot \bar{N} \neq \mathbf{0}$ cohesive zone model

$\llbracket \varphi \rrbracket \neq \mathbf{0}, \llbracket P \rrbracket \cdot \overline{\boldsymbol{N}} \neq \mathbf{0}$

elasticity theory together with continuum damage; $\mathbf{d}$ loadings "parallel" and "normal" to the interface causing both opening across and in-plane deformation of the interface, accounting for interface damage, modeled by interface elasticity theory together with cohesive zone and continuum damage (the current work). The different color of the interface represents different interface material properties as compared to those of the bulk. (Color figure online)

erences therein. The effect of interface energetics, e.g. for inclusions, and the size-dependent elastic state of the material has been widely investigated recently for instance in [7-9, 21,23-27,34, 41, 78-80,94] and references therein.

Nonetheless, the interface elasticity theory suffers from the fact that the interface behavior remains elastic regardless of the strain level at the interface. This issue can be addressed by extending interface elasticity, such that it also accounts for damage along the coherent interface (see Fig. 1c). Hence, this interface is labeled inelastic. Note that in this work interface inelasticity is only damage-type. In principle, one can derive an interface damage model as a natural counterpart to the bulk damage model. This is motivated by the fact that the well-established interface elasticity theory is essentially the interface counterpart of bulk elasticity theory. In this work, the interface tangential damage variable $\bar{D}_{\|}$is a function of the interface deformation gradient $\overline{\boldsymbol{F}}$ which measures the interface tangential stretch or compression (see Fig. 2b). 
(a)

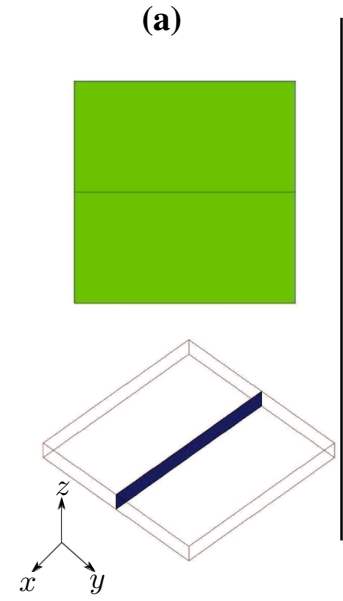

undeformed configuration

$\mathrm{w} /$ straight interface (b)

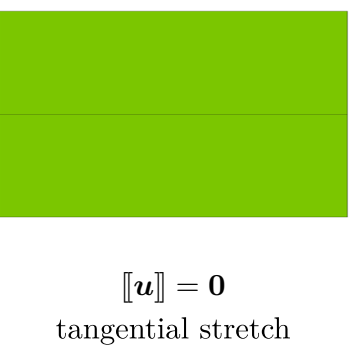

interface elasticity

coherent interface (c)
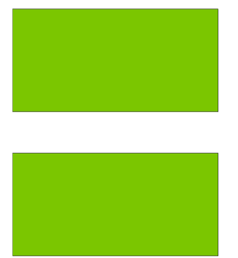

$\llbracket \boldsymbol{u}_{\mathrm{s}} \rrbracket=\mathbf{0}$

$\llbracket u_{\mathrm{n}} \rrbracket \neq \mathbf{0}$

$\mathrm{w} /$ normal opening

$w /$ shear opening

$\llbracket u_{\mathrm{s}} \rrbracket \neq \mathbf{0}$

$\llbracket u_{\mathrm{n}} \rrbracket=\mathbf{0}$

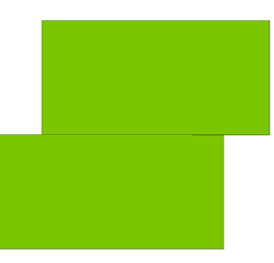

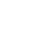

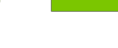

$\mathrm{w} /$

hear \& normal opening

$\mathrm{w} /$ tangential deformation

(current contribution)

classical CZM classical CZM
Fig. 2 Illustration of interface elasticity and cohesive zone model. Starting with the reference configuration (a) and applying load at the right boundary (b), top boundary (c), top half of the right boundary (d), and the top half of the right boundary at an angle of $45^{\circ}$ (e), a classical cohesive interface shows no mechanical in-plane resistance $(\mathbf{c})-(\mathbf{d})$, whereas an energetic interface is tangentially resistant to deformation

Analogous to the bulk material, processes such as fracture can also occur on the (in)elastic interface creating a non-coherent interface for which a well established cohesive zone model can be utilized (see Fig. 1a). Classically, a noncoherent non-energetic interface behavior is often described by cohesive zone models, with the constitutive response being represented by a traction-separation law. Therefore the interface traction and material degradation, measured by a cohesive damage variable $\bar{D}_{\nVdash}$, are functions of the displacement jump vector. We label such an interface cohesive. Section 1.1 briefly reviews this approach.

However, there exist some limitations with the classical CZM. The first main shortcoming is that it only treats nonenergetic interfaces, neglecting the material existing between the two sides of the interface (see Figs. 1a and 2c-d). Noting that the interface discussed here represents a thin layer of material with mechanical properties independent of the surrounding bulk, it is crucial to take into account the mechanical response of the material in between the two sides of the noncoherent interface (see Fig. 1d). This is pronounced at the nanometer scale where the surface/interface area to the bulk volume ratio is significant. The second issue with the classical cohesive zone model stems from the fact it can not capture the interface mechanical response if the loading conditions do not cause any form of opening displacement (see Fig. 2b). This is due to the fact that the classical CZM lacks any deformation resistance against tangential stretch of the interface. Recall that an interface here represents one or several layers of material and thus its mechanical response must not solely depend on whether or not there exists a displacement (b). An interface could deform and degrade tangentially without facing any displacement jump (b). An interface could also encounter displacement jump and cohesive degradation without any tangential deformation (c)-(d). The current contribution couples interface (in)elasticity and CZM to model (in)elastic (energetic) non-coherent interfaces (e). (Color figure online)

jump across the interface. We also point out that the tangential deformation of the interface mid-plane and shear/sliding displacement jump across the interface are two very different phenomena (compare Fig. $2 b$ with d). The former is measured in terms of a second-order superficial deformation gradient $\overline{\boldsymbol{F}}$ and the latter in terms of the displacement jump vector $\llbracket \boldsymbol{u} \rrbracket$ with $\llbracket \boldsymbol{u}_{\mathrm{s}} \rrbracket$ and $\llbracket \boldsymbol{u}_{\mathrm{n}} \rrbracket$ being its shear and normal components. The former then causes interface stress on the tangential midplane of the interface resulting in the superficial second-order Piola stress tensor $\overline{\boldsymbol{P}}$, while the latter causes traction $\boldsymbol{T}$, a vector quantity, ${ }^{3}$ across the interface. To induce stress on the tangential plane of the interface one needs to apply deformation on the elastic interface, whereas a cohesive interface is existent if and only if there is some form of opening (normal or shear) across the interface. Finally the material degradation of a cohesive interface (see [72] for a review of such models) does not include the tangential degradation of the elastic interface, since an interface could possibly undergo a large deformation before it even begins to encounter any displacement jump (see Fig. 2 and refer to Sect. 1.2 for further discussions).

In the current work, the in-between interface material is represented by a zero-thickness fictitious mid-plane ${ }^{4}$ for which the extension of the interface elasticity theory to

\footnotetext{
3 Also note the differences between the units of interface stress and traction that are $\mathrm{N} / \mathrm{mm}$ and $\mathrm{N} / \mathrm{mm}^{2}$, respectively. The unit of length in this work is $\mathrm{mm}$.

4 The term "mid-plane" is only valid in the case of non-coherency on the interface.
} 
(a)

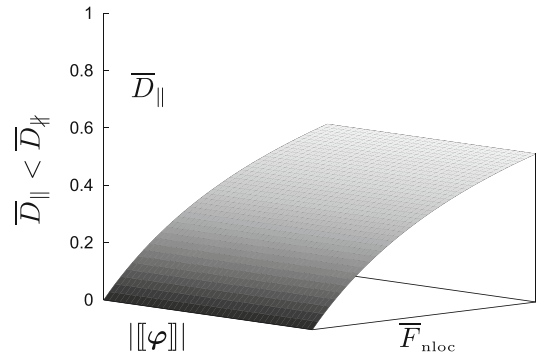

(b)

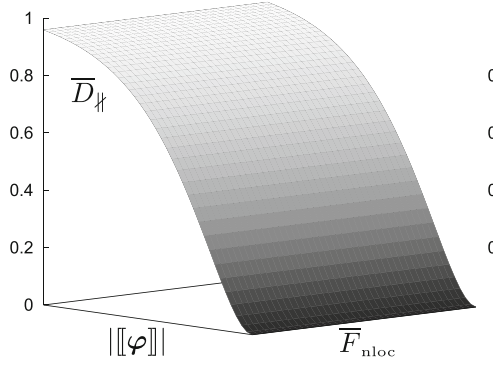

(c)

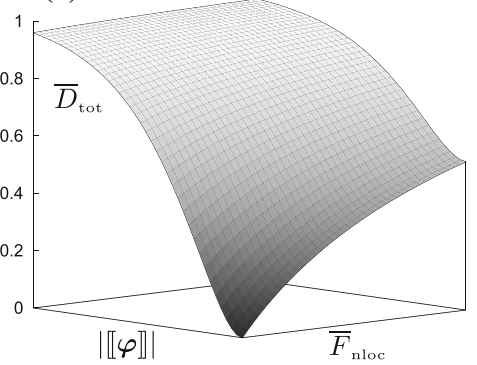

Fig. 3 Tangential damage profile $\bar{D}_{\|}(\mathbf{a})$, cohesive damage profile $\bar{D}_{\Varangle}$ (b), and total damage profiles $\bar{D}_{\text {tot }}(\mathbf{c})$, with respect to effective opening displacement $|\llbracket \varphi \rrbracket|$ and non-local equivalent distortion $\bar{F}_{\text {nloc }}$. The total damage variable $0 \leq \bar{D}_{\text {tot }} \leq 1$ is related to $\bar{D}_{\|}$and $\bar{D}_{\nVdash}$ in the form $\bar{D}_{\text {tot }}=\bar{D}_{\|}+\bar{D}_{\Varangle}-\bar{D}_{\|} \bar{D}_{\nVdash}$. In this example, the attained maximum values of $\bar{D}_{\|}, \bar{D}_{\nVdash}$ and $\bar{D}_{\text {tot }}$ are $0.6,1$ and 1, respectively. The total out-of-plane material break down $\left(\bar{D}_{\nVdash}=1\right)$ causes the total interface material breakdown $\left(\bar{D}_{\text {tot }}=1\right)$, although the interface mid-plane is not yet fully damaged $\left(\bar{D}_{\|}=0.6<1\right)$. Alternatively, the total in-plane material break down $\left(\bar{D}_{\|}=1\right)$ results in the total interface material breakdown $\left(\bar{D}_{\text {tot }}=1\right)$, regardless of the value taken by $\bar{D}_{\nVdash}$

cal support was provided by [65] for the model of [40]. A potential-based cohesive zone model was proposed by [62], which was generalized by $[73,92,93]$ to account for shear decohesion, tensile decohesion and irreversibility [62, see also]. The mode-mixity was introduced in [86] and used by [15]. Recently a small-strain thermodynamically-based fictitious crack model was formulated by [66]. A finite element irreversible mixed-mode formulation was given by $[64,70]$.

The majority of cohesive zone laws in the literature are either polynomial [86], piece-wise linear [87], exponential [92], rigid-linear cohesive zone laws [11] or some combinations thereof, whereby these are either intrinsic or extrinsic $[52,88]$.

Various cohesive constitutive relations accounting for dissipation and hysteresis were presented in the works of [63,64, $76,89]$. A consistent formulation of a crack/interface model applicable to finite deformations was given for instance by $[4,31,37,56,59,85]$. The large-deformation cohesive models can be divided into two groups depending on weather the opening displacement is defined in the material configuration $[60,85]$ or in the spatial configuration $[37,56,64,89,90$, see for instance]. In a recent contribution [67] provided a largedeformation cohesive zone framework satisfying the angular momentum balance. Furthermore [68] extended the work in [67] to include energetic interfaces.

In the field of composite delamination many authors e.g. $[1,3,5,10,16,18,19,53,57,77]$ employed the continuum damage mechanics approach to model the degradation of non-coherent, cohesive interface elements. A formulation for a non-local damage-type cohesive zone model was provided by $[14,42]$. Within a thermomechanical framework $[32,35$, $69,91]$ studied the effects of the degradation of the cohesive interface on thermal properties. The coupling of an interface damage model and friction is provided in $([2,16,55,74,75]$, among others) using cohesive zone models. 


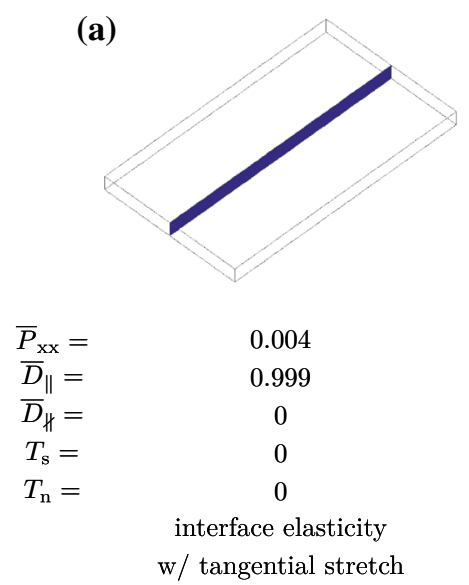

(a)

w/ tangential stretch (b)

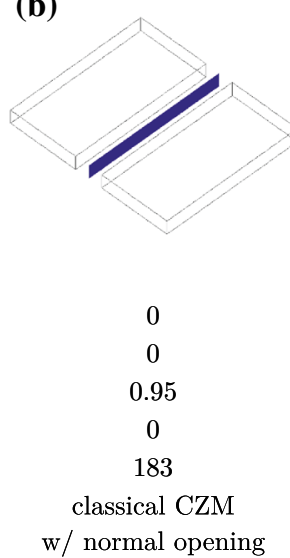

(c)
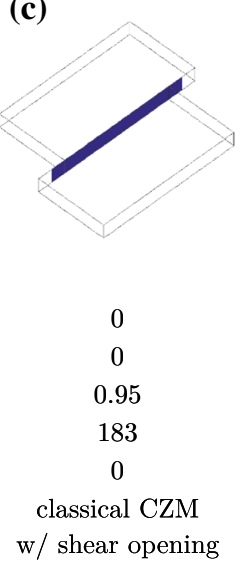

(d)

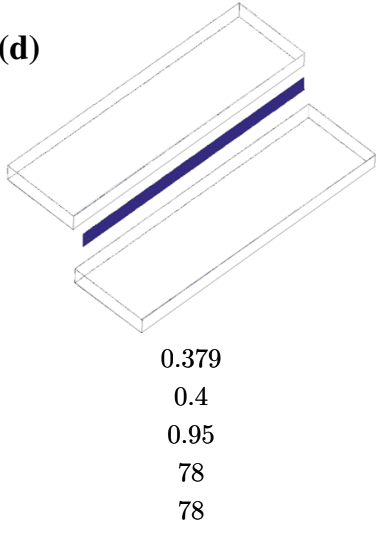

$$
\begin{gathered}
\text { CZM + interface elasticity } \\
\text { w/ tangential stretch } \\
+ \text { normal \& shear opening } \\
\text { (current work) }
\end{gathered}
$$

Fig. 4 Strip with straight interface. The undeformed configuration is given in Fig. 2. The maximum extension is $d_{\max }^{\mathrm{p}}=1 \mathrm{~mm}$. Dimensions are in mm (see Fig. 7). Result a shows in-plane (tangential) stretch and degradation of a coherent interface. A force is applied parallel to the interface, uniformly on the right boundary while the left boundary is fixed. Interface inelasticity can be used to study this case. Result $\mathbf{b}$ shows an interface undergone a normal opening only and cohesive degradation. A force is applied on the top boundary while fixing the bottom half of the domain. A CZM is used to model such an interface. The force in $\mathbf{c}$ is applied parallel to the interface, uniformly on the top half

\subsection{Tangential deformation versus shear sliding}

Following the discussion in the introduction, we provide an example in Fig. 4, where different boundary conditions are applied to a strip with a straight interface. The undeformed configuration is illustrated in Fig. 2a. It is important to recall that the interface in this work represents one or several layers of thin materials which undergo deformation, inelastic and/or fracture processes. As stated before, the model developed here couples a classical cohesive zone model and interface inelasticity.

In Fig. 4a, a force is applied on the right boundary of the domain, parallel to the interface. Under this condition the interface only experiences tangential stretch (deformation) resulting in stress on the interface plane $\left(\bar{P}_{\mathrm{xx}} \neq 0\right)$ whereby the interface remains coherent (no displacement jump across the interface). Due to such stretch, the tangential degradation of the coherent interface initiates and evolves until complete material breakdown $\left(\bar{D}_{\|} \approx 1\right)$. This type of interface can only be modeled by an interface (in)elasticity theory.

In Fig. $4 \mathrm{~b}$ a force is applied on the top boundary while fixing the bottom boundary. This condition results in a normal opening and normal traction $T_{\mathrm{n}}$ across the interface and no superficial Piola stress $\overline{\boldsymbol{P}}$ on the interface mid-plane. Note that the cohesive degradation is fully developed $\left(\bar{D}_{\nVdash} \approx\right.$ of the right boundary while the bottom half of the strip is fixed. A shear opening and cohesive degradation is the result of the above boundary condition. A CZM is used to model such an interface. The force in $\mathbf{d}$ is applied analogously to (c), here however, at an angle of $45^{\circ}$. A mixedmode opening and traction, in-plane stress on the interface mid-plane, cohesive and tangential interface degradations are observed. The current work models this type of generalized interface behavior. Shear and normal traction, the xx component of interface Piola stress, cohesive and tangential damage variable are denoted by $T_{\mathrm{s}}$ and $T_{\mathrm{n}}, \bar{P}_{\mathrm{xx}}, \bar{D}_{\nVdash}$ and $\bar{D}_{\|}$, respectively

1) while no tangential (in-plane) degradation is detected $\left(\bar{D}_{\|}=0\right)$.

In Fig. 4c we apply the force only on the top half of the right boundary while the bottom half of the domain is fixed. Under these circumstances, a shear opening and shear traction $T_{\mathrm{S}}$ across the interface is observed. Note that here, too, only the cohesive interface degradation is present, i.e. $\bar{D}_{\nVdash}>0$.

In Fig. 4d the force is only exerted on the top half of the right boundary at an angle of $45^{\circ}$, while the bottom half of the domain are fixed. Under these circumstances, a mixed-mode opening and traction $T_{\mathrm{s}}, T_{\mathrm{n}}$ across the interface, together with a superficial Piola stress $\overline{\boldsymbol{P}}$ on the interface mid-plane are observed. To study such a complex case, we use the model developed in this work: a coupling of interface elasticity, damage modelling and CZM. Note that now interface traction, stress, and cohesive and tangential degradations are present, i.e. $T_{\mathrm{s}} \neq 0, T_{\mathrm{n}} \neq 0, \overline{\boldsymbol{P}} \neq \mathbf{0}, \bar{D}_{\nVdash}>0$ and $\bar{D}_{\|}>0$.

\subsection{Organization of this manuscript}

This manuscript is organized as follows. First the notation and certain key concepts are briefly introduced. Section 2 summarizes the kinematics and the governing balance equations of non-linear continuum mechanics. A non-local continuum damage model on the mid-plane of the interface, a 
Table 1 List of important terminologies and notations

\begin{tabular}{llll}
\hline Bulk & & Interface & \\
\hline $\boldsymbol{F}$ & Bulk deformation gradient & $\overline{\boldsymbol{F}}$ & Interface deformation gradient \\
$\boldsymbol{\varphi}$ & Bulk deformation map & $\overline{\boldsymbol{\varphi}}$ & Interface deformation map \\
$\boldsymbol{X}$ & Bulk material coordinates & $\overline{\boldsymbol{X}}$ & Interface material coordinates \\
$\boldsymbol{x}$ & Bulk spatial coordinates & $\overline{\boldsymbol{x}}$ & Interface spatial coordinates \\
$\boldsymbol{N}$ & Bulk material normal to surface & $\overline{\boldsymbol{N}}$ & Interface material normal to interface \\
$\boldsymbol{n}$ & Bulk spatial normal to surface & $\overline{\boldsymbol{n}}$ & Interface spatial normal to interface \\
$\boldsymbol{P}$ & Bulk nominal Piola stress & $\overline{\boldsymbol{P}}$ & Interface nominal Piola stress \\
$\Psi$ & Bulk nominal free energy & $\bar{\Psi}$ & Interface nominal free energy \\
\hline Only interface & & & \\
\hline $\boldsymbol{\varphi}^{ \pm}$ & & & \\
$\llbracket \boldsymbol{\varphi} \rrbracket$ & Deformation maps of \pm sides & $\boldsymbol{x}^{ \pm}$ & Spatial coordinates of \pm sides \\
$\tilde{\boldsymbol{n}}^{ \pm}$ & Deformation jump & $\overline{\boldsymbol{P}}_{0}$ & Effective Piola stress \\
$\boldsymbol{T}$ & Spatial normal to interface boundary & $\widetilde{\boldsymbol{N}}$ & Material normal to interface boundary \\
$\bar{\Psi}_{\|}$ & Nominal cohesive traction & $\overline{\mathbb{A}}_{\|}$ & Interface nominal elasticity tensor \\
$\bar{\Psi}_{\|}^{0}$ & Nominal free energy & $\bar{\Psi}_{\sharp}$ & Nominal cohesive free energy \\
$\bar{D}_{\|}$ & Effective free energy & $\bar{\Psi}_{\sharp}^{0}$ & Effective cohesive free energy \\
$\bar{D}_{\text {tot }}$ & Tangential damage parameters & $\bar{D}_{\sharp}$ & Cohesive damage parameters \\
$\bar{F}_{0}$ & Total damage parameter & $\bar{F}_{\text {nloc }}$ & Non-local equivalent distortion \\
$\delta_{\mathrm{c}}$ & Elastic limit & $\bar{F}_{\text {max }}$ & Maximum attained $\bar{F}_{\text {nloc }}$ \\
\hline & Critical opening displacement & $\sigma_{\mathrm{c}}$ & Critical cohesive traction \\
\hline
\end{tabular}

cohesive zone model on the non-coherent interface and the coupling of these two models are derived in Sect. 3. A specific interface Helmholtz energy and its arguments together with a specific interface cohesive potential and its arguments are proposed. The coupling of the tangential and cohesive damage variables and the necessary requirements for such a coupling are presented. Thermodynamically consistent constitutive relations are then determined. A numerical framework that encompasses interface elasticity coupled with non-local damage and a cohesive zone model on the interface is established in Sect. 4. The framework includes the weak formulation of the governing equations, the corresponding finite element implementation and the derivation of the consistent stiffness matrices. A series of numerical examples, based on the finite element approximation of the weak form, is presented in Sect. 5 to elucidate the theory. Section 6 concludes this work.

\subsection{Notation and definitions}

Direct notation is adopted throughout. Occasional use is made of index notation, the summation convention for repeated indices being implied. The three-dimensional Euclidean space is denoted by $\mathbb{E}^{3}$. The scalar product of two vectors $\boldsymbol{a}$ and $\boldsymbol{b}$ is denoted by $\boldsymbol{a} \cdot \boldsymbol{b}=[\boldsymbol{a}]_{i}[\boldsymbol{b}]_{i}$. The scalar product of two second-order tensors $\boldsymbol{A}$ and $\boldsymbol{B}$ is denoted by $\boldsymbol{A}: \boldsymbol{B}=[\boldsymbol{A}]_{i j}[\boldsymbol{B}]_{i j}$. The composition of two second-order tensors $\boldsymbol{A}$ and $\boldsymbol{B}$, denoted by $\boldsymbol{A} \cdot \boldsymbol{B}$, is a secondorder tensor with components $[\boldsymbol{A} \cdot \boldsymbol{B}]_{i j}=[\boldsymbol{A}]_{i m}[\boldsymbol{B}]_{m j}$. The vector product of two vectors $\boldsymbol{a}$ and $\boldsymbol{b}$ is denoted by $\boldsymbol{a} \times \boldsymbol{b}$ with $[\boldsymbol{a} \times \boldsymbol{b}]_{k}=[\boldsymbol{\varepsilon}]_{i j k}[\boldsymbol{a}]_{i}[\boldsymbol{b}]_{j}$ where $\boldsymbol{\varepsilon}$ denotes the permutation (Levi-Civita) tensor. The non-standard product of a fourth-order tensor $\mathbb{C}$ and a vector $\boldsymbol{b}$ is defined by $[\boldsymbol{b}: \mathbb{C}]_{i k l}=[\mathbb{C}]_{i j k l}[\boldsymbol{b}]_{j}$. The action of a second-order tensor $\boldsymbol{A}$ on a vector $\boldsymbol{a}$ is given by $[\boldsymbol{A} \cdot \boldsymbol{a}]_{i}=[\boldsymbol{A}]_{i j}[\boldsymbol{a}]_{j}$. The standard product of a fourth-order tensor $\mathbb{C}$ and a second-order tensor $\boldsymbol{A}$ is defined by $[\mathbb{C}: \boldsymbol{A}]_{i j}=[\mathbb{C}]_{i j k l}[\boldsymbol{A}]_{k l}$. The dyadic product of two vectors $\boldsymbol{a}$ and $\boldsymbol{b}$ is a second-order tensor $\boldsymbol{D}=\boldsymbol{a} \otimes \boldsymbol{b}$ with $[\boldsymbol{D}]_{i j}=[\boldsymbol{a}]_{i}[\boldsymbol{b}]_{j}$. Two non-standard dyadic products of two second-order tensors $\boldsymbol{A}$ and $\boldsymbol{B}$ are the fourth-order tensors $[\boldsymbol{A} \bar{\otimes} \boldsymbol{B}]_{i j k l}=[\boldsymbol{A}]_{i k}[\boldsymbol{B}]_{j l}$ and $[\boldsymbol{A} \otimes \boldsymbol{B}]_{i j k l}=[\boldsymbol{A}]_{i l}[\boldsymbol{B}]_{j k}$. The average and jump of a quantity $\{\bullet\}$ over the interface are defined by $\{\{\bullet\}\}\}=\frac{1}{2}\left[\{\bullet\}^{+}+\{\bullet\}^{-}\right]$and $\llbracket\{\bullet\} \rrbracket=\{\bullet\}^{+}-\{\bullet\}^{-}$ respectively. All over-lined quantities correspond to the interface. Table 1 gathers a list of notations frequently used in this manuscript.

\section{Problem definition}

This section summarizes the kinematics of non-linear continuum mechanics including material generalized interfaces and introduces the notation adopted here. Further details on the kinematics of deformable interfaces can be found in [49]. 

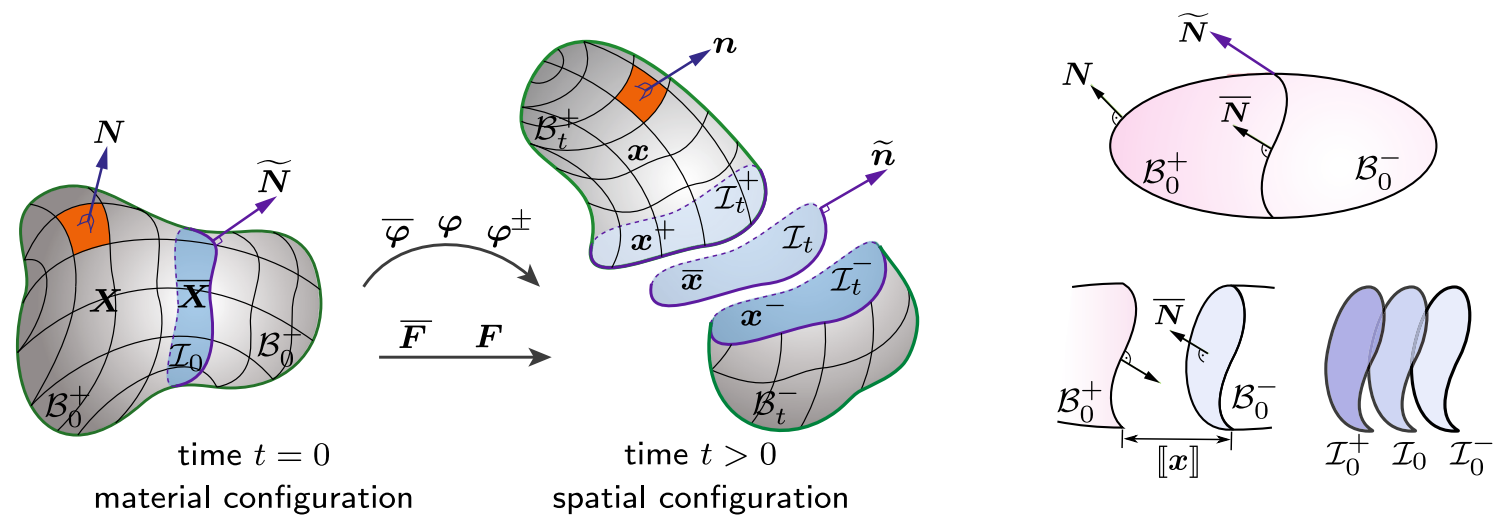

Fig. 5 The bulk domain $\mathcal{B}_{0}$, the bulk subdomains $\mathcal{B}_{0}^{ \pm}$, the interface $\mathcal{I}_{0}$, the two sides of the interface $\mathcal{I}_{0}^{ \pm}$and the unit normals to the surface $N$, mid-plane of the interface $\bar{N}$, and boundary of the interface $\widetilde{N}$, all defined in the material configuration. The bulk, interface and the two sides of interface deformation maps, denoted as $\varphi, \bar{\varphi}$ and $\varphi^{ \pm}$, respectively, map the material configuration to the spatial configuration at time $t$. The bulk domain $\mathcal{B}_{t}$, the bulk subdomains $\mathcal{B}_{t}^{ \pm}$, the interface

Consider a continuum body $\mathscr{B}$ that takes the material configuration $\mathcal{B}_{0} \subset \mathbb{E}^{3}$ at time $t=0$, and the spatial configuration $\mathcal{B}_{t}$ at $t>0$, as depicted in Fig. 5 . The body $\mathscr{B}$ is partitioned into two disjoint subdomains, $\mathcal{B}_{0}^{+}$and $\mathcal{B}_{0}^{-}$, by an interface $\mathcal{I}_{0}$, thus the bulk is defined by $\mathcal{B}_{0}:=\mathcal{B}_{0}^{+} \cup \mathcal{B}_{0}^{-}$, with reference placements of material particles labeled $X$. The two sides of the interface $\mathcal{I}_{0}$ are denoted $\mathcal{I}_{0}^{+}:=\partial \mathcal{B}_{0}^{+} \cap \mathcal{I}_{0}$ and $\mathcal{I}^{-}:=\partial \mathcal{B}_{0}^{-} \cap \mathcal{I}_{0}$. The material particles on the interface are labeled by $\overline{\boldsymbol{X}}$. The outward unit normal to $\partial \mathcal{B}_{0}$ is denoted $N$. The outward unit normal to the boundary of the interface $\partial \mathcal{I}_{0}$, tangent to the interface $\mathcal{I}_{0}$ is denoted $\widetilde{N}$. The unit normal to $\mathcal{I}_{0}$ is denoted $\bar{N}$ whose direction is conventionally taken to point from the negative side of the interface to the positive side. The spatial counterparts of the various unit normals are $\boldsymbol{n}, \tilde{\boldsymbol{n}}$ and $\overline{\boldsymbol{n}}$, respectively. The deformation maps of the bulk, and the negative and positive sides of the interface are denoted $\varphi, \varphi^{-}$and $\varphi^{+}$, respectively. The restriction of the motion $\varphi$ to the interface is defined by $\bar{\varphi}:=\{\{\varphi\}\}$. The current placements of particles in the bulk and on the two sides of the interface are denoted $\boldsymbol{x}$ and $\boldsymbol{x}^{\mp}$ where the spatial placement of particles on the mid-plane of the interface are designated as $\overline{\boldsymbol{x}}:=\{\{\boldsymbol{x}\}$. Note that $\llbracket \boldsymbol{\varphi} \rrbracket \neq \mathbf{0}$ and $\llbracket \boldsymbol{x} \rrbracket \neq$ $\mathbf{0}$ denote the opening displacement across the generalized interface.

The bulk and the rank-deficient interface deformation gradients are respectively defined by

$\boldsymbol{F}(\boldsymbol{X}, t):=\operatorname{Grad} \varphi(\boldsymbol{X}, t)$ and

$\overline{\boldsymbol{F}}(\overline{\boldsymbol{X}}, t):=\overline{\operatorname{Grad}} \overline{\boldsymbol{\varphi}}(\overline{\boldsymbol{X}}, t)$.

The interface gradient and divergence operators are respectively defined by
$\mathcal{I}_{t}$ and its two sides $\mathcal{I}_{t}^{ \pm}$, the unit normals to the surface $\boldsymbol{n}$, interface $\overline{\boldsymbol{n}}$, and boundary of the interface $\widetilde{\boldsymbol{n}}$, all defined in the spatial configuration. The deformation jump across the interface in the spatial configuration is denoted by $\llbracket \boldsymbol{x} \rrbracket$. The interface unit normal is pointing from the negative to the positive side of the interface. The bulk and rank-deficient interface deformation gradients are $\boldsymbol{F}$ and $\overline{\boldsymbol{F}}$, respectively

$$
\begin{aligned}
\overline{\operatorname{Grad}}\{\overline{\boldsymbol{\bullet}}\} & :=\operatorname{Grad}\{\overline{\boldsymbol{\bullet}}\} \cdot \overline{\boldsymbol{I}} \text { and } \\
\overline{\operatorname{Div}}\{\bar{\bullet}\} & :=\overline{\operatorname{Grad}}\{\overline{\boldsymbol{\bullet}}\}: \overline{\boldsymbol{I}} \text { with } \overline{\boldsymbol{I}}:=\boldsymbol{I}-\overline{\boldsymbol{N}} \otimes \overline{\boldsymbol{N}} .
\end{aligned}
$$

where $\overline{\boldsymbol{I}}$ and $\boldsymbol{I}$ denote the interface and bulk unit tensors. Their spatial counterparts are denoted $\overline{\boldsymbol{i}}$ and $\boldsymbol{i}$. Finally the bulk and interface Jacobians are denoted by $J:=\operatorname{det} \boldsymbol{F}>0$ and $\bar{J}:=\overline{\operatorname{det}} \overline{\boldsymbol{F}}>0$ respectively, with $\overline{\operatorname{det}}\{\bullet\}$ denoting the area determinant [84].

Equilibrium conditions in the bulk and on a generalized interface together with associated boundary conditions are listed in Table 2 (see $[48,49]$, for further details).

A detailed derivation of the balance of forces and moments on the generalized interface is presented in "Balance of forces and moments on the interface" Appendix section. The interface Piola stress tensor $\overline{\boldsymbol{P}}$ is a superficial ${ }^{6}$ tensor field possessing the property $\overline{\boldsymbol{P}} \cdot \overline{\boldsymbol{N}}=\mathbf{0}$. In the absence of $\overline{\boldsymbol{b}}^{\mathrm{p}}$, the jump of traction across the interface equates with the negative divergence of the interface stress tensor. Therefore, the classical traction continuity across the interface $(\llbracket \boldsymbol{P} \rrbracket \cdot \overline{\boldsymbol{N}}=\mathbf{0})$ no longer holds. The balance of moments on the interface can also be rewritten as $[\{\boldsymbol{P}\} \cdot \overline{\boldsymbol{N}}] \otimes \llbracket \boldsymbol{r} \rrbracket+\overline{\boldsymbol{F}} \cdot \overline{\boldsymbol{P}}^{\mathrm{t}}$, which is symmetric (see "Balance of forces and moments on the interface" Appendix section for further details). Note that $\overline{\boldsymbol{P}}$ represents the nominal surface stress on the mid-plane of the interface.

Furthermore, for the brevity of exposition no material degradation is here considered in the bulk.

\footnotetext{
$\overline{6}$ The superficiality of the interface Piola stress tensor is a classical assumption of interface elasticity theory. Recently, [47] have proven that this condition is the consequence of a first-order continuum theory.
} 
Table 2 Localized force and moment balances in the bulk and on the generalized interface in the material configuration

\begin{tabular}{|c|c|c|c|}
\hline \multirow[t]{2}{*}{ Force balance } & $\operatorname{Div} \boldsymbol{P}+\boldsymbol{b}^{\mathrm{p}}=\mathbf{0}$ & in $\mathcal{B}_{0}$ & $\widehat{\boldsymbol{b}}^{\mathrm{p}}=\boldsymbol{P} \cdot \boldsymbol{N} \quad$ on $\partial \mathcal{B}_{0}{ }^{\mathrm{N}}$ \\
\hline & $\overline{\operatorname{Div}} \overline{\boldsymbol{P}}+\overline{\boldsymbol{b}}^{\mathrm{p}}+\llbracket \boldsymbol{P} \rrbracket \cdot \overline{\boldsymbol{N}}=\mathbf{0}$ & on $\mathcal{I}_{0}$ & $\widetilde{\boldsymbol{b}}^{\mathrm{p}}=\overline{\boldsymbol{P}} \cdot \widetilde{\boldsymbol{N}} \quad$ on $\partial \mathcal{I}_{0}^{\mathrm{N}}$ \\
\hline \multirow[t]{2}{*}{ Moment balance } & $\boldsymbol{P} \cdot \boldsymbol{F}^{\mathrm{t}}=\boldsymbol{F} \cdot \boldsymbol{P}^{\mathrm{t}}$ & in $\mathcal{B}_{0}$ & \\
\hline & $\llbracket r \rrbracket \times[\{\boldsymbol{P}\}] \cdot \bar{N}]+\varepsilon:\left[\bar{F} \cdot \overline{\boldsymbol{P}}^{\mathrm{t}}\right]=\mathbf{0}$ & on $\mathcal{I}_{0}$ & \\
\hline$b^{\mathrm{p}}$ & Force vector per unit volume & $\widehat{b}^{\mathrm{p}}$ & Surface traction per unit area \\
\hline$\overline{\boldsymbol{b}}^{\mathrm{p}}$ & Force vector per unit area & $\widetilde{\boldsymbol{b}}^{\mathrm{p}}$ & Curve traction per unit length \\
\hline$r$ & Position vector & $\varepsilon$ & Permutation tensor \\
\hline
\end{tabular}

The notation $\{\bullet\}^{\mathrm{p}}$ is to distinguish prescribed quantities. The notation $\{\bullet\}^{\mathrm{t}}$ is the transposition operator

\section{Damage modeling for generalized interfaces}

In this section first an isotropic non-local in-plane damage model in terms of integral averaging is briefly presented for large deformations on the interface. This model takes into account the in-plane degradation of the interface. Second, an irreversible finite-deformation cohesive zone model is reviewed to account for the material decohesion across the interface. Finally, the above models are coupled by their corresponding damage variables (tangential $\bar{D}_{\|}$and cohesive $\left.\bar{D}_{\nvdash}\right)$ in a specific manner so that material degradation in any of the directions (along or across the interface) affects the material stiffness in the other direction.

\subsection{In-plane interface damage model}

Damage is here modeled by introducing an internal variable $\bar{D}_{\|}$. This variable then relates the interface nominal (damaged) Piola stress tensor $\overline{\boldsymbol{P}}$ to the interface effective (undamaged) stress tensor $\overline{\boldsymbol{P}}_{0}$ through a reduction factor $\left[1-\bar{D}_{\|}\right]$, i.e. $\overline{\boldsymbol{P}}=\left[1-\bar{D}_{\|}\right] \overline{\boldsymbol{P}}_{0}$.

To proceed, a Helmholtz energy ${ }^{7}$ is considered containing the following arguments

$\bar{\Psi}_{\|}\left(\overline{\boldsymbol{F}}, \bar{D}_{\|}, \bar{\kappa}\right)=\left[1-\bar{D}_{\|}\right] \bar{W}_{\|}^{0}(\overline{\boldsymbol{F}})+\int_{0}^{\bar{\kappa}} \overline{\mathcal{H}}\left(\bar{\kappa}^{*}\right) \mathrm{d} \bar{\kappa}^{*}$,

with $\bar{D}_{\|} \in[0,1]$ where $\bar{W}_{\|}^{0}(\overline{\boldsymbol{F}})$ is the interface effective (undamaged) Helmholtz energy and $\overline{\mathcal{H}}(\bar{\kappa})$ denotes a monotonically increasing function depending on the internal variable $\bar{\kappa}$. Differentiating Eq. (3) with respect to time and particularizing the interface Clausius-Plank inequality one finds

$$
\begin{aligned}
\overline{\mathcal{D}}_{\text {int }}= & {\left[\overline{\boldsymbol{P}}-\left[1-\bar{D}_{\|}\right] \frac{\partial \bar{W}_{\|}^{0}(\overline{\boldsymbol{F}})}{\partial \overline{\boldsymbol{F}}}\right]: \dot{\boldsymbol{F}} } \\
& +\bar{W}_{\|}^{0}(\overline{\boldsymbol{F}}) \dot{\bar{D}}_{\|}-\overline{\mathcal{H}}(\bar{\kappa}) \dot{\bar{\kappa}} \geq 0 .
\end{aligned}
$$

7 The integral term in Eq. (3) is introduced in analogy to that of [81, Sect. 1.3.3] and denotes the energy storage in the material due to the accumulation of microscopic defects.
Therefore, the interface nominal Piola stress tensor $\overline{\boldsymbol{P}}$ and the reduced dissipation $\overline{\mathcal{D}}_{\text {red }}$ are expressed as

$\overline{\boldsymbol{P}}=\left[1-\bar{D}_{\|}\right] \overline{\boldsymbol{P}}_{0} \quad$ with $\quad \overline{\boldsymbol{P}}_{0}=\frac{\partial \bar{W}_{\|}^{0}(\overline{\boldsymbol{F}})}{\partial \overline{\boldsymbol{F}}}$,

$\overline{\mathcal{D}}_{\text {red }}=\bar{Y} \dot{\bar{D}}_{\|}-\overline{\mathcal{H}}(\bar{\kappa}) \dot{\bar{\kappa}} \geq 0$ with $\bar{Y}=-\frac{\partial \bar{\Psi}_{\|}}{\partial \bar{D}_{\|}}$,

where $\bar{Y}=\bar{W}_{\|}^{0}(\overline{\boldsymbol{F}})$, driving the damage evolution, is the thermodynamic force conjugate to the interface damage variable $\bar{D}$. Next a damage condition $\bar{\Upsilon}$ is motivated as [83]

$\bar{\Upsilon}(\bar{Y}, \overline{\mathcal{H}})=\bar{v}(\bar{Y})-\overline{\mathcal{H}}(\bar{\kappa}) \leq 0$,

with $\bar{v}$ being a monotonically increasing function. The KuhnTucker conditions then read

$\bar{\Upsilon}(\bar{Y}, \overline{\mathcal{H}}) \leq 0, \quad \bar{\lambda}^{*} \geq 0, \quad \bar{\lambda}^{*} \bar{\Upsilon}(\bar{Y}, \overline{\mathcal{H}})=0$,

with $\bar{\lambda}^{*}$ being the consistency parameter. Now by choosing $\bar{v}(\bullet)=\overline{\mathcal{H}}(\bullet)$, and defining the change of variables $\bar{F}_{\max }:=f(\bar{\kappa})$ and $\bar{F}_{\text {nloc }}:=f(\bar{Y})$ and assuming $f$ to be a monotonically increasing function with the property $f(0)=0$, an alternative damage condition to Eq. (7) takes the form

$\bar{\phi}\left(\bar{F}_{\text {nloc }}, \bar{F}_{\max }\right)=\bar{F}_{\text {nloc }}-\bar{F}_{\max } \leq 0$ with

$\bar{F}_{\text {nloc }}\left(\overline{\boldsymbol{x}}_{\mathrm{r}}\right)=\int_{\mathcal{I}_{0}} \bar{\omega}\left(\overline{\boldsymbol{x}}_{\mathrm{r}}, \overline{\boldsymbol{x}}_{\mathrm{s}}\right) \bar{F}_{\text {loc }}\left(\boldsymbol{x}_{\mathrm{s}}\right) \mathrm{d} A$ and

$\bar{F}_{\text {loc }}:=\sqrt{2 \bar{Y} / \bar{E}}$,

where $\bar{F}_{\text {max }}(t)=\max _{s \in[0, t]}\left\{\bar{F}_{0},\left.\quad \bar{F}_{\text {nloc }}\right|_{s}\right\}, \bar{F}_{0}$ is the damage threshold, $\bar{F}_{\text {loc }}$ is the local equivalent distortion, and $\bar{E}$ is the interface Young's modulus. Note that the tangential damage variable is a function of $\bar{F}_{\text {max }}$, i.e. $\bar{D}_{\|}=\overline{\mathscr{D}}\left(\bar{F}_{\text {max }}\right)$. The evolution of damage occurs when $\bar{\phi}=0$, which characterizes the damage surface. In Eq. $(9)_{2}, \bar{\omega}\left(\overline{\boldsymbol{x}}_{\mathrm{r}}, \overline{\boldsymbol{x}}_{\mathrm{s}}\right)$ is a given non-local weight function depending on the geodesic dis- 
(a)

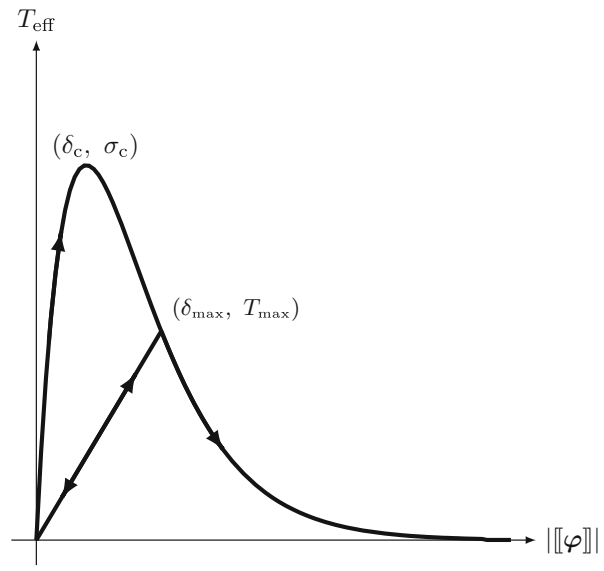

Fig. 6 Exponential traction-separation law (a) and stress vs. equivalent distortion with exponential softening on the interface (b). The parameters $\delta_{\mathrm{c}}$ and $\sigma_{\mathrm{c}}$ are critical opening and traction respectively. The maximum attained effective opening displacement is denoted by $\delta_{\max }$

tance ${ }^{8} \bar{r}=\left\|\overline{\boldsymbol{x}}_{\mathrm{r}}-\overline{\boldsymbol{x}}_{\mathrm{s}}\right\|_{\mathcal{I}}$ between the source point $\overline{\boldsymbol{x}}_{\mathrm{s}}$ and the receiver point $\overline{\boldsymbol{x}}_{\mathrm{r}}$. Note that in Eq. (9) 2 , the integral extends on the lower-dimensional manifold $\mathcal{I}_{0}$. In order to resolve the issue of alteration of a uniform field in the vicinity of the boundary, the weight function is scaled leading to

$$
\begin{aligned}
\bar{\omega}\left(\overline{\boldsymbol{x}}_{\mathrm{r}}, \overline{\boldsymbol{x}}_{\mathrm{s}}\right)=\frac{\bar{\omega}_{0}(\bar{r})}{\int_{\mathcal{I}_{0}} \bar{\omega}_{0}(\bar{r}) \mathrm{d} A} \text { with } \\
\bar{\omega}_{0}(\bar{r})=\left\{\begin{array}{lll}
{\left[1-\frac{\bar{r}^{2}}{\bar{R}^{2}}\right]^{2}} & \text { if } & |\bar{r}| \leq \bar{R}, \\
0 & \text { if } \quad|\bar{r}| \geq \bar{R},
\end{array}\right.
\end{aligned}
$$

where $\bar{\omega}_{0}(\bar{r})$ is a non-negative and monotonically decreasing (for $\bar{r} \geq 0$ ) piecewise polynomial bell-shaped function. The interface interaction radius is denoted by $\bar{R}$.

Finally, a smooth function to relate the damage variable $\bar{D}_{\|}$to the history variable $\bar{F}_{\text {max }}$ with resulting exponential softening law is chosen as follows:

$$
\bar{D}_{\|}=\left\{\begin{array}{lll}
0 & \text { if } & \bar{F}_{\max } \leq \bar{F}_{0} \\
1-\frac{\bar{F}_{0}}{\bar{F}_{\text {max }}} \exp \left(-\frac{\bar{F}_{\text {max }}-\bar{F}_{0}}{\bar{F}_{\mathrm{f}}-\bar{F}_{0}}\right) & \text { if } & \bar{F}_{\max } \geq \bar{F}_{0},
\end{array}\right.
$$

where $\bar{F}_{\mathrm{f}}$ affects the ductility of the response (see Fig. $6 \mathrm{~b}$ ).

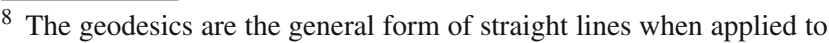
curved, three-dimensional interfaces. The minimal geodesics in differential geometry are the shortest distance paths between two points on a interface.
}

(b)

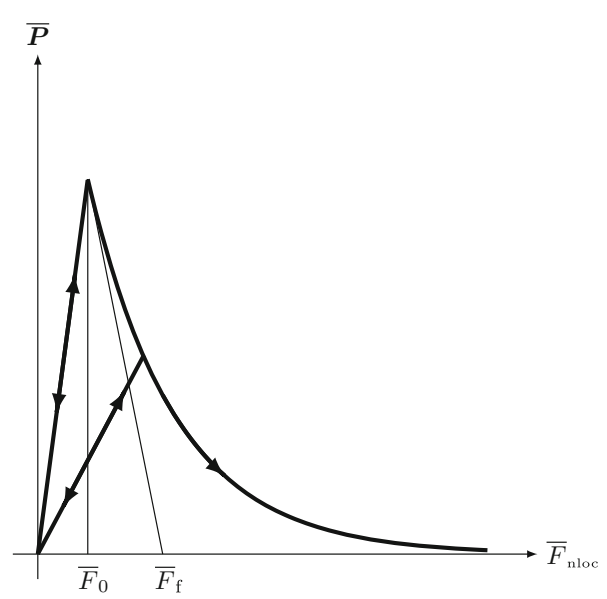

and $T_{\max }$ is the effective traction evaluated at $\delta_{\max }$. The parameters $\bar{F}_{0}$ and $\bar{F}_{\mathrm{f}}$ are the interface critical equivalent distortion and ductility response

\subsection{Out-of-plane interface damage model}

The focus in this section is on the derivation of an irreversible finite-deformation cohesive zone model $^{9}$ on the non-coherent interface. A classical traction-separation law can be obtained (see [56], for further details) from a potential which in the absence of internal variables takes the form

$\bar{\Psi}_{\nVdash}^{\sharp 0}=\exp (1) \sigma_{\mathrm{c}} \delta_{\mathrm{c}}\left[1-\left[1+\frac{|\llbracket \boldsymbol{\varphi} \rrbracket|}{\delta_{\mathrm{c}}}\right] \exp \left(-|\llbracket \boldsymbol{\varphi} \rrbracket| / \delta_{\mathrm{c}}\right)\right]$.

Consequently by taking the derivative of cohesive energy Eq. (12) with respect to the opening displacement vector $\llbracket \varphi \rrbracket$ one can find the traction vector as follows $[56,64]$, if $|\llbracket \varphi \rrbracket|=\delta_{\max }$ and $\quad|\llbracket \dot{\varphi} \rrbracket| \geq 0$, then,

$\boldsymbol{T}_{0}=\underbrace{\exp (1) \sigma_{\mathrm{c}} \frac{|\llbracket \boldsymbol{\varphi} \rrbracket|}{\delta_{\mathrm{c}}} \exp \left(-|\llbracket \boldsymbol{\varphi} \rrbracket| / \delta_{\mathrm{c}}\right)}_{T_{\mathrm{eff}}} \frac{\llbracket \boldsymbol{\llbracket \varphi}}{|\llbracket \boldsymbol{\varphi} \rrbracket|}$,

where $\delta_{\mathrm{c}}$ is a characteristic opening displacement at which $T_{\text {eff }}$ reaches a critical value denoted by $\sigma_{\mathrm{c}}$ and $\delta_{\max }$ is the maximum attained effective opening displacement introduced to take into account the irreversibility of the model (see Fig. 6a). The constitutive relation in the case of unloading takes the form $[11,64]$

\footnotetext{
${ }^{9}$ Since in the current cohesive zone model the traction vector is co-linear with the displacement jump vector, the balance of angular momentum on the interface is fulfilled. See [67] for further details.
} 
$\boldsymbol{T}_{0}=\frac{T_{\max }}{\delta_{\max }} \llbracket \boldsymbol{\varphi} \rrbracket \quad$ if $\quad|\llbracket \boldsymbol{\varphi} \rrbracket|<\delta_{\max } \quad$ or $\quad|\llbracket \dot{\boldsymbol{\varphi}} \rrbracket|<0$,

where $T_{\max }$ is calculated by evaluating $T_{\text {eff }}$ in Eq. (13) at $\delta_{\max }$.

The linearization of the cohesive traction with respect to the jump term, i.e. the cohesive tangent, is give in the case of loading as

$$
\begin{aligned}
& \mathcal{T}_{0}=-\frac{1}{\delta_{\mathrm{c}}} \exp (1) \sigma_{\mathrm{c}} \frac{|\llbracket \boldsymbol{} \rrbracket|}{\delta_{\mathrm{c}}} \exp \left(-|\llbracket \boldsymbol{\varphi} \rrbracket| / \delta_{\mathrm{c}}\right) \frac{\llbracket \boldsymbol{\varphi} \rrbracket}{|\llbracket \boldsymbol{\varphi} \rrbracket|} \otimes \frac{\llbracket \boldsymbol{| \varphi} \rrbracket}{|\llbracket \boldsymbol{\varphi} \rrbracket|} \\
& +\exp (1) \sigma_{\mathrm{c}} \frac{1}{\delta_{\mathrm{c}}} \exp \left(-|\llbracket \boldsymbol{\varphi} \rrbracket| / \delta_{\mathrm{c}}\right) \frac{\llbracket \boldsymbol{\varphi} \rrbracket}{|\llbracket \boldsymbol{\varphi} \rrbracket|} \otimes \frac{\llbracket \boldsymbol{|} \rrbracket}{|\llbracket \varphi \rrbracket|} \\
& +\frac{\exp (1)}{|\llbracket \varphi \rrbracket|} \sigma_{\mathrm{c}} \frac{|\llbracket \varphi \rrbracket|}{\delta_{\mathrm{c}}} \exp \left(-|\llbracket \varphi \rrbracket| / \delta_{\mathrm{c}}\right) \\
& {\left[I-\frac{\llbracket \varphi \rrbracket}{|\llbracket \varphi \rrbracket|} \otimes \frac{\llbracket \varphi \rrbracket}{|\llbracket \varphi \rrbracket|}\right] .}
\end{aligned}
$$

Correspondingly in the case of unloading the cohesive tangent reads

$\mathcal{T}_{0}=\frac{T_{\max }}{\delta_{\max }} \boldsymbol{I}$

Although the current cohesive model is not based on an explicit definition of a cohesive damage variable, as required by the coupling in Sect. 3.3, we shall define a cohesive damage parameter as follows

$D_{\nVdash}=\frac{\bar{\Psi}_{\nVdash}^{\sharp 0}\left(\delta_{\max }\right)}{G_{\mathrm{c}}}$ with $0 \leq D_{\nVdash} \leq 1$,

where the critical energy release rate $G_{\mathrm{c}}$ is obtained by

$$
\begin{aligned}
G_{\mathrm{c}} & =\int_{0}^{\infty} T_{\text {eff }} \mathrm{d}|\llbracket \varphi \rrbracket| \text { using } T_{\text {eff }} \text { in Eq. (13) } \Longrightarrow \\
G_{\mathrm{c}} & =\exp (1) \sigma_{\mathrm{c}} \delta_{\mathrm{c}} .
\end{aligned}
$$

Finally by taking the time derivative of Eq. (17) one obtains the evolution of the cohesive damage variable as follows

$\dot{D}_{\nVdash}=\frac{1}{G_{\mathrm{c}}} \frac{\partial \bar{\Psi}_{\nVdash}^{\nVdash 0}\left(\delta_{\max }\right)}{\partial \delta_{\max }} \dot{\delta}_{\max } \geq 0$, with

$\dot{\delta}_{\max }= \begin{cases}\dot{\delta}_{\text {eff }} & \text { if } \quad|\llbracket \boldsymbol{\varphi} \rrbracket|=\delta_{\max } \text { and }|\llbracket \dot{\varphi} \rrbracket| \geq 0 \\ 0 & \text { otherwise, }\end{cases}$

which is consistent with the irreversibility of damage [64]. In addition, relation $(19)_{2}$ states that the cohesive interfacial response during a cyclic unloading-reloading does not change as long as the opening displacement does not exceed its formerly attained maximum value upon reloading.

\subsection{Coupling of tangential damage and cohesive model}

The focus of this section is on the coupling of material degradations along and across the interface. As stated earlier the tangential damage variable $\bar{D}_{\|}$is introduced to account for in-plane degradation of the interface. The cohesive damage $\bar{D}_{\Varangle}$ is defined to measure the decohesion across the noncoherent interface. Now in what follows the coupling of the two models (continuum damage and cohesive zone) using the above damage variables is presented.

First a total damage variable $\bar{D}_{\text {tot }}$ is introduced as follows

$\bar{D}_{\text {tot }}=\bar{D}_{\|}+\bar{D}_{\Varangle}-\bar{D}_{\|} \bar{D}_{\Varangle}$ where $0 \leq \bar{D}_{\text {tot }} \leq 1$,

in order to meet the following requirements

if $\left\{\begin{array}{l}\bar{D}_{\|}=0 \text { or } \bar{D}_{\sharp}=0, \\ \bar{D}_{\|}=1 \text { or } \bar{D}_{\sharp}=1,\end{array} \quad\right.$ then $\bar{D}_{\text {tot }}=\max \left\{\bar{D}_{\|}, \bar{D}_{\nVdash}\right\}$.

Note that the first requirement in Eq. (21) 1 ensures that the initiation of damage in any direction also triggers the interface damage in the remaining direction, for which the associated damage variable might still be zero. The second condition in Eq. (21) 1 guarantees that the cohesive or tangential total breakdown results in the total breakdown of the interface material.

Having defined the total damage variable $\bar{D}_{\text {tot }}$, the arguments of the interface total free energy $\bar{\Psi}$ are now selected as

$\bar{\Psi} \equiv \bar{\Psi}\left(\overline{\boldsymbol{F}}, \llbracket \boldsymbol{\varphi} \rrbracket, \bar{D}_{\text {tot }}\right)=\underbrace{\bar{\Psi}_{\|}\left(\overline{\boldsymbol{F}}, \bar{D}_{\text {tot }}\right)}_{\text {tangential }}+\underbrace{\bar{\Psi}_{\nVdash}\left(\llbracket \varphi \rrbracket, \bar{D}_{\text {tot }}\right)}_{\text {cohesive }}$,

$\bar{\Psi} \equiv\left[1-\bar{D}_{\text {tot }}\right] \bar{\Psi}^{0}=\underbrace{\left[1-\bar{D}_{\text {tot }}\right] \bar{\Psi}_{\|}^{0}}_{\text {tangential }}+\underbrace{\left[1-\bar{D}_{\text {tot }}\right] \bar{\Psi}_{\nVdash}^{0}}_{\text {cohesive }}$,

where $\bar{\Psi}_{\|}^{0}$ and $\bar{\Psi}_{\nVdash}^{0}$ are tangential and cohesive effective interface energies, respectively. Moreover $\left[1-\bar{D}_{\text {tot }}\right]$ and $\bar{\Psi}_{\|}^{0}$ are defined as

$\left[1-\bar{D}_{\text {tot }}\right]=\left[1-\bar{D}_{\|}\right]\left[1-\bar{D}_{\nmid}\right]$,

and

$\bar{\Psi}_{\|}^{0}(\overline{\boldsymbol{F}}, \bar{\kappa})=\bar{W}_{\|}^{0}(\overline{\boldsymbol{F}})+\int_{0}^{\bar{\kappa}} \overline{\mathcal{H}}^{0}\left(\bar{\kappa}^{*}\right) \mathrm{d} \bar{\kappa}^{*}$,

respectively, where $\overline{\mathcal{H}}^{0}$ is a monotonically increasing function. In summary the interface free energy $\bar{\Psi}$ is composed of two terms: the tangential free energy $\bar{\Psi}_{\|}$and the cohesive free energy $\bar{\Psi}_{\nVdash}$, defined respectively, by 


$$
\begin{aligned}
\bar{\Psi}_{\|}\left(\overline{\boldsymbol{F}}, \bar{D}_{\text {tot }}\right)= & {\left[1-\bar{D}_{\text {tot }}\right] \bar{W}_{\|}^{0}(\overline{\boldsymbol{F}}) } \\
& +\left[1-\bar{D}_{\text {tot }}\right] \int_{0}^{\bar{\kappa}} \overline{\mathcal{H}}^{0}\left(\bar{\kappa}^{*}\right) \mathrm{d} \bar{\kappa}^{*} \text { and } \\
\bar{\Psi}_{\nVdash}\left(\llbracket \boldsymbol{\varphi} \rrbracket, \bar{D}_{\text {tot }}\right)= & {\left[1-\bar{D}_{\text {tot }}\right] \bar{\Psi}_{\nVdash}^{0}(\llbracket \boldsymbol{\varphi} \rrbracket) } \\
= & {\left[1-\bar{D}_{\|}\right] \bar{\Psi}_{\nVdash}^{\nVdash 0}(\llbracket \varphi \rrbracket), }
\end{aligned}
$$

We should point out that the definition of $\bar{\Psi}_{\sharp}^{\sharp 0}$ implicitly includes the effect of $\left[1-\bar{D}_{\Varangle}\right]$, see Eq. (12) and the related discussions.

To better understand the nature of the above proposed form for the interface free energy $\bar{\Psi}$ one can study two extreme cases: (i) a cohesive (non-coherent and non-energetic) interface, Eq. (12), with $\bar{\Psi}_{\|}=0$ and (ii) an energetic (coherent) interface, Eq. (3), with $\bar{\Psi}_{\Varangle}=0$. Note that for these extreme cases, as the respective damage variables, i.e $\bar{D}_{\Varangle}$ and $\bar{D}_{\|}$ evolve, the corresponding energies are reduced (dissipated) which correspond to a damage-type cohesive zone model and an interface continuum damage approach, respectively. Regarding the interface in this work which is cohesive and energetic, two sources of interface energy are consequently required meaning that now the total interface free energy is the sum over these partial energies. However, the summation is now performed by multiplying the partial energies by the total reduction factor given in Eq. (23) so that when the interface material is degraded cohesively and/or tangentially, all the partial energies are reduced accordingly to make sure that the interface total material is damaged, see also Eq. (21) and the related discussions. This idea stems from the fact it must not be possible to encounter cohesive degradation while the in-plane interface properties remain intact and vice versa. For further clarification let's now assume the following two loading case:

- $|\llbracket \varphi \rrbracket| \gg \delta_{\text {c }}$, thus $\bar{D}_{\Varangle} \approx 0$, and $\bar{F}_{\text {nloc }}<\bar{F}_{0}$, thus $\bar{D}_{\Varangle}=0$. These assumptions result in $\bar{\Psi}=0$ since $\bar{\Psi}_{\sharp}^{\sharp 0}=0$, and $\bar{\Psi}_{\|} \approx 0$ because $\left[1-\bar{D}_{\text {tot }}\right] \approx 0$, despite the fact that $\bar{W}_{\|}^{0}>0$.

$-\bar{F}_{\text {nloc }} \gg \bar{F}_{0}$, thus $\bar{D}_{\|} \approx 1$, and $|\llbracket \varphi \rrbracket|<\delta_{\mathrm{c}}$, thus $\bar{D}_{\Varangle}=0$. These assumptions result in $\bar{\Psi}=0$ since $\bar{\Psi}_{\|} \approx 0$, and $\bar{\Psi}_{\Varangle} \approx 0$ because $\left[1-\bar{D}_{\|}\right] \approx 0$, despite the fact that $\bar{\Psi}_{\sharp}^{\sharp^{0}}>0$.

Since the interface mid-plane represents the material between the two sides of the cohesive interface, this plane must also be degraded with the evolution of cohesive damage (case 1 above). The other case is equally necessary. If the interface mid-plane undergoes a material degradation due to a large tangential deformation, the cohesive stiffness must as well be reduced (case 2 above). The energy formulation given in
Eq. (25) realizes both of the above and all the situation that fall in between.

Differentiating Eq. (22) with respect to time, using Eq. (25)

$$
\begin{aligned}
\dot{\bar{\Psi}}= & {\left[1-\bar{D}_{\text {tot }}\right]\left[\frac{\partial \bar{W}_{\|}^{0}(\overline{\boldsymbol{F}})}{\partial \overline{\boldsymbol{F}}}: \dot{\overline{\boldsymbol{F}}}+\frac{\partial \bar{\Psi}_{\nVdash}^{0}(\llbracket \boldsymbol{\varphi} \rrbracket)}{\partial \llbracket \boldsymbol{\varphi} \rrbracket} \cdot \llbracket \dot{\boldsymbol{\varphi} \rrbracket}\right] } \\
& -\left[1-\bar{D}_{\nVdash}\right] \underbrace{\left[\bar{W}_{\|}^{0}(\overline{\boldsymbol{F}}) \dot{\bar{D}}_{\|}-\left[1-\bar{D}_{\|}\right] \overline{\mathcal{H}}^{0}(\bar{\kappa}) \dot{\bar{\kappa}}\right]}_{\overline{\mathcal{D}}_{\mathrm{red}}} \\
& -\left[1-\bar{D}_{\|}\right] \dot{\bar{D}}_{\nVdash} \bar{W}_{\|}^{0}(\overline{\boldsymbol{F}}) \\
& -\left[\dot{\bar{D}}_{\|}\left[1-\bar{D}_{\nVdash]}\right]+\dot{\bar{D}}_{\nVdash}\left[1-\bar{D}_{\|}\right]\right] \int_{0}^{\bar{\kappa}} \overline{\mathcal{H}}^{0}\left(\bar{\kappa}^{*}\right) \mathrm{d} \bar{\kappa}^{*} \\
& -\left[\dot{\bar{D}}_{\|}\left[1-\bar{D}_{\nVdash]}\right]+\dot{\bar{D}}_{\nVdash}\left[1-\bar{D}_{\|}\right]\right] \bar{\Psi}_{\nVdash}^{0}(\llbracket \boldsymbol{\varphi} \rrbracket) \geq 0,
\end{aligned}
$$

and particularizing the interface Clausius-Plank inequality and using Eq. (26) one finds

$$
\begin{aligned}
\overline{\mathcal{D}}_{\text {int }}= & {\left[\overline{\boldsymbol{P}}-\left[1-\bar{D}_{\text {tot }}\right] \frac{\partial \bar{W}_{\|}^{0}(\overline{\boldsymbol{F}})}{\partial \overline{\boldsymbol{F}}}\right]: \dot{\overline{\boldsymbol{F}}} } \\
& +\left[\boldsymbol{T}-\left[1-\bar{D}_{\text {tot }}\right] \frac{\partial \bar{\Psi}_{\nVdash}^{0}(\llbracket \boldsymbol{\varphi} \rrbracket)}{\partial \llbracket \boldsymbol{\varphi} \rrbracket}\right] \cdot \llbracket \dot{\boldsymbol{\varphi} \rrbracket}+\overline{\mathcal{D}}_{*}, \text { with, } \\
\overline{\mathcal{D}}_{*}= & {\left[1-\bar{D}_{\nVdash}\right] \overline{\mathcal{D}}_{\text {red }}+\left[1-\bar{D}_{\|}\right] \dot{\bar{D}}_{\nVdash} \bar{W}_{\|}^{0}(\overline{\boldsymbol{F}}) } \\
& +\left[\dot{\bar{D}}_{\|}\left[1-\bar{D}_{\nVdash]}\right]+\dot{\bar{D}}_{\nVdash}\left[1-\bar{D}_{\|}\right]\right] \int_{0}^{\bar{\kappa}} \overline{\mathcal{H}}^{0}\left(\bar{\kappa}^{*}\right) \mathrm{d} \bar{\kappa}^{*} \\
& +\left[\dot{\bar{D}}_{\|}\left[1-\bar{D}_{\nVdash]}\right]+\dot{\bar{D}}_{\nVdash}\left[1-\bar{D}_{\|}\right]\right] \bar{\Psi}_{\nVdash}^{0}(\llbracket \boldsymbol{} \rrbracket) \geq 0 .
\end{aligned}
$$

Thus, the interface nominal Piola stress tensor $\overline{\boldsymbol{P}}$ and the cohesive traction vector $\boldsymbol{T}$ are expressed as

$\overline{\boldsymbol{P}}=\left[1-\bar{D}_{\text {tot }}\right] \overline{\boldsymbol{P}}_{0}$ and $\quad \boldsymbol{T}=\left[1-\bar{D}_{\text {tot }}\right] \frac{\partial \bar{\Psi}_{\nVdash}^{0}(\llbracket \boldsymbol{\varphi} \rrbracket)}{\partial \llbracket \boldsymbol{\varphi} \rrbracket}$

The positive-semi-definiteness of Eq. (27) is fulfilled noting that

$\left\{\begin{array}{l}0 \leq \bar{D}_{\nVdash} \leq 1 \quad \text { and } \overline{\mathcal{D}}_{\text {red }} \geq 0 \text { from Eq. (6) }, \\ 0 \leq \bar{D}_{\|} \leq 1, \quad \bar{W}_{\|}^{0} \geq 0, \quad \int_{0}^{\bar{\kappa}} \overline{\mathcal{H}}^{0}\left(\bar{\kappa}^{*}\right) \mathrm{d} \bar{\kappa}^{*} \geq 0, \\ \dot{\bar{D}}_{\nVdash} \geq 0 \text { from Eq. (19), } \\ \left.\dot{\bar{D}}_{\|} \geq 0 \text { from Eq. (6) }\right)_{1} \text { and } \bar{\Psi}_{\nVdash}^{0} \geq 0 .\end{array}\right.$ 
Finally the interface tangent tensor for the local damage model and cohesive tangent are now obtained as

$$
\begin{aligned}
\overline{\mathbb{A}} & =\frac{\partial \overline{\boldsymbol{P}}}{\partial \overline{\boldsymbol{F}}} \\
& = \begin{cases}\overline{\mathbb{A}}_{\text {sect }}-\left[1-\bar{D}_{\sharp}\right] \frac{\overline{\mathscr{D}}^{\prime}}{\bar{E}_{\bar{F}_{\text {loc }}}} \overline{\mathbb{P}}_{0} & \text { if } \bar{\phi}=0 \text { and } \dot{\bar{F}}_{\text {loc }}>0, \\
\overline{\mathbb{A}}_{\text {sect }} & \text { otherwise, }\end{cases}
\end{aligned}
$$

with $\overline{\mathbb{A}}_{0}=\partial \overline{\boldsymbol{P}}_{0} / \partial \overline{\boldsymbol{F}}, \overline{\mathbb{A}}_{\text {sect }}=\left[1-\bar{D}_{\text {tot }}\right] \overline{\mathbb{A}}_{0}$ being the secant tangent tensor, $\overline{\mathscr{D}}^{\prime}=\partial \overline{\mathscr{D}}\left(\bar{F}_{\max }\right) / \partial \bar{F}_{\max }, \overline{\mathbb{P}}_{0}=\overline{\boldsymbol{P}}_{0} \otimes \overline{\boldsymbol{P}}_{0}$, and

$\mathcal{T}=\frac{\partial \boldsymbol{T}}{\partial \llbracket \varphi \rrbracket}=\left[1-\bar{D}_{\|}\right] \mathcal{T}_{0}$

respectively. The derivation of the non-local interface tangent tensor will be discussed in Sect. 4.

\section{Computational framework}

The purpose of this section is to establish a numerical framework for generalized interfaces. Deriving the weak form and spatial (finite element) discretizations will be presented next.

\subsection{Weak form}

To derive the mechanical weak form, the localized force balance equations in the bulk and on the interface given in Table 2 are tested (from the left) with vector valued test functions $\delta \varphi \in \mathscr{H}^{1}\left(\mathcal{B}_{0}\right)$ and $\delta \bar{\varphi} \in \mathscr{H}^{1}\left(\mathcal{I}_{0}\right)$, respectively. The result is then integrated over the corresponding domains in the material configuration. Using the bulk and interface diverg ence theorems (see "Extended divergence theorem" Appendix section) and the orthogonality properties of the interface Piola stress measures, the weak form of the balance of linear momentum is

$$
\begin{aligned}
& \int_{\mathcal{B}_{0}} \boldsymbol{P}: \operatorname{Grad} \delta \boldsymbol{\varphi} \mathrm{d} V+\int_{\mathcal{I}_{0}} \overline{\boldsymbol{P}}: \overline{\operatorname{Grad}} \delta \overline{\boldsymbol{\varphi}} \mathrm{d} A \\
& -\int_{\mathcal{I}_{0}} \underbrace{[\{\boldsymbol{P}\}] \cdot \overline{\boldsymbol{N}}]}_{\boldsymbol{T}} \cdot \llbracket \delta \boldsymbol{\varphi} \rrbracket \mathrm{d} A-\int_{\mathcal{B}_{0}} \delta \boldsymbol{\varphi} \cdot \boldsymbol{b}^{\mathrm{p}} \mathrm{d} V \\
& -\int_{\mathcal{I}_{0}} \delta \overline{\boldsymbol{\varphi}} \cdot \overline{\boldsymbol{b}}^{\mathrm{p}} \mathrm{d} A-\int_{\partial \mathcal{B}_{0}^{\mathrm{N}}} \delta \boldsymbol{\varphi} \cdot \widehat{\boldsymbol{b}}_{\mathrm{N}}^{\mathrm{p}} \mathrm{d} A \\
& -\int_{\partial \mathcal{I}_{0}^{\mathrm{N}}} \delta \overline{\boldsymbol{\varphi}} \cdot \widetilde{\boldsymbol{b}}_{\mathrm{N}}^{\mathrm{p}} \mathrm{d} L=0, \\
& \forall \delta \boldsymbol{\varphi} \in \mathscr{H}^{1}\left(\mathcal{B}_{0}\right), \forall \delta \overline{\boldsymbol{\varphi}} \in \mathscr{H}^{1}\left(\mathcal{I}_{0}\right) \quad \text { with } \delta \overline{\boldsymbol{\varphi}}=\left\{\left.\{\delta \boldsymbol{\varphi}\}\right|_{\mathcal{I}_{0} .}\right.
\end{aligned}
$$

A detailed derivation of the mechanical weak form is presented in "Weak form of the balance of forces" Appendix section.

\subsection{Finite element implementation}

In order to apply the finite element method to the present problem, the weak form Eq. (32) is discretized. The discretization is carried in space by means of the finite element method (see [46,51], for further details).

In order to have a straightforward and efficient implementation of the finite element method, the interface elements are chosen to be consistent with the bulk elements. For example, if the bulk is discretized using triquadratic elements, then biquadratic interface elements are used. This choice has the advantage that common facet of two adjacent bulk elements can be regarded as an interface element.

The geometry of the bulk, interface and the deformation jump over the interface are approximated as a function of the natural coordinates $\xi \in[-1,1]^{3}$ and $\bar{\xi} \in[-1,1]^{2}$ assigned to the bulk and the interface, respectively, using standard interpolations according to the isoparametric concept as follows

$$
\begin{aligned}
& \left.\boldsymbol{X}\right|_{\mathcal{B}_{0}^{\beta}} \approx \boldsymbol{X}^{\mathrm{h}}(\boldsymbol{\xi})=\sum_{i=1}^{n_{\mathrm{nB}}} N^{i}(\boldsymbol{\xi}) \boldsymbol{X}^{i}, \\
& \left.\overline{\boldsymbol{X}}\right|_{\mathcal{I}_{0}^{\gamma}} \approx \overline{\boldsymbol{X}}^{\mathrm{h}}(\bar{\xi})=\sum_{i=1}^{n_{\mathrm{nI}}} \bar{N}^{i}(\bar{\xi}) \overline{\boldsymbol{X}}^{i}, \\
& \left.\boldsymbol{\varphi}\right|_{\mathcal{B}_{0}^{\beta}} \approx \boldsymbol{\varphi}^{\mathrm{h}}(\boldsymbol{\xi})=\sum_{i=1}^{n_{\mathrm{nB}}} N^{i}(\boldsymbol{\xi}) \boldsymbol{\varphi}^{i}, \\
& \left.\overline{\boldsymbol{\varphi}}\right|_{\mathcal{I}_{0}^{\gamma}} \approx \overline{\boldsymbol{\varphi}}^{\mathrm{h}}(\bar{\xi})=\sum_{i=1}^{n_{\mathrm{nI}}} \bar{N}^{i}(\bar{\xi}) \overline{\boldsymbol{\varphi}}^{i}, \\
& \left.\llbracket \boldsymbol{x}\right|_{\mathcal{I}_{0}^{\gamma}} \approx \llbracket \boldsymbol{x} \rrbracket^{\mathrm{h}}(\bar{\xi})=\sum_{i=1}^{n_{\mathrm{nI}}} \bar{N}^{i}(\bar{\xi}) \llbracket \boldsymbol{x} \rrbracket^{i}, \\
& \left.\llbracket \boldsymbol{\varphi}\right|_{\mathcal{I}_{0}^{\gamma}} \approx \llbracket \boldsymbol{\varphi} \rrbracket^{\mathrm{h}}(\bar{\xi})=\sum_{i=1}^{n_{\mathrm{nI}}} \bar{N}^{i}(\bar{\xi}) \llbracket \boldsymbol{\varphi} \rrbracket^{i},
\end{aligned}
$$

where $\mathcal{B}_{0}^{\beta}$ and $\mathcal{I}_{0}^{\gamma}$ are the $\beta$ th and $\gamma$ th element in the bulk and on the interface, respectively. The shape functions of the bulk and interface elements at a local node $i$ are denoted by $N^{i}$ and $\bar{N}^{i}$, respectively. Every bulk and interface element consists of $n_{\mathrm{nB}}$ and $n_{\mathrm{nI}}$ nodes, respectively.

Next, the fully discrete form of mechanical residual vector ${ }^{\text {tot }} \mathbf{R}^{I}$ associated with the global node $I$ is defined by ${ }^{10}$

\footnotetext{
10 In what follows, for the sake of brevity, homogeneous Neumann boundary conditions are assumed and the body forces are omitted and hence, some integrals vanish. The integrals are standard and require no additional care for a generalized interface.
} 


$$
\begin{aligned}
{\left[{ }^{\text {tot }} \mathbf{R}^{I}\right]:=} & \int_{\mathcal{B}_{0}} \boldsymbol{P} \cdot \operatorname{Grad} N^{I} \mathrm{~d} V \\
& +\int_{\mathcal{I}_{0}} \overline{\boldsymbol{P}} \cdot \overline{\operatorname{Grad}} \bar{N}^{I} \mathrm{~d} A-\left[\mp \int_{\mathcal{I}_{0}} \boldsymbol{T} \bar{N}^{I} \mathrm{~d} A\right] .
\end{aligned}
$$

Note that the total nodal mechanical (momentum) residual ${ }^{\text {tot }} \mathbf{R}^{I}$ consists of contributions from both the bulk and the interface, i.e. ${ }^{\text {tot }} \mathbf{R}^{I}=\mathbf{R}^{I}+{ }^{\text {tot }} \overline{\mathbf{R}}^{I}$ where the total nodal interface residual ${ }^{\text {tot }} \overline{\mathbf{R}}^{I}$ is composed of

$$
\begin{gathered}
\overline{\mathbf{R}}^{I}=\underbrace{\int_{\mathcal{I}_{0}} \overline{\boldsymbol{P}} \cdot \overline{\operatorname{Grad}} \bar{N}^{I} \mathrm{~d} A}_{\text {interface in-plane }} \text { and } \\
\overline{\mathbf{R}}^{I^{ \pm}}=\underbrace{-\left[\mp \int_{\mathcal{I}_{0}} \boldsymbol{T} \bar{N}^{I} \mathrm{~d} A\right]}_{\text {interface out-of-plane }} .
\end{gathered}
$$

The global momentum residual vector takes the form

$$
{ }^{\text {tot }} \mathbf{R}=\left[\begin{array}{c}
{ }^{\text {tot }} \mathbf{R}^{1} \\
\vdots \\
{ }^{\text {tot }} \mathbf{R}^{I} \\
\vdots \\
{ }^{\text {tot }} \mathbf{R}^{n_{\mathrm{Bn}}}
\end{array}\right]=\left[\begin{array}{c}
\mathbf{R}^{1} \\
\vdots \\
\mathbf{R}^{I} \\
\vdots \\
\mathbf{R}^{n_{\mathrm{Bn}}}
\end{array}\right]+\left[\begin{array}{c}
\overline{\mathbf{R}}^{1} \\
\vdots \\
\overline{\mathbf{R}}^{I} \\
\vdots \\
\overline{\mathbf{R}}^{n_{\mathrm{In}}}
\end{array}\right]+\left[\begin{array}{c}
\overline{\mathbf{R}}^{1^{ \pm}} \\
\vdots \\
\overline{\mathbf{R}}^{I^{ \pm}} \\
\vdots \\
\overline{\mathbf{R}}^{n_{\mathrm{In}}^{ \pm}}
\end{array}\right]
$$

where $n_{\mathrm{Bn}}$ and $n_{\text {In }}$ denote the total number of bulk and interface nodes. The summation operator implies the (conventional) residual assembly of finite element method.

The fully-discrete coupled non-linear system of governing equations can be stated as follows

${ }^{\text {tot }} \mathbf{R}(\mathbf{d}) \stackrel{!}{=} \mathbf{0}, \quad$ with $\quad \mathbf{d}=\left[\begin{array}{lllll}\varphi^{1} & \ldots & \varphi^{I} & \ldots & \varphi^{n_{\mathrm{Bn}}}\end{array}\right]$

where $\mathbf{d}$ is the unknown global vector of spatial coordinates. To solve (36), a Newton-Raphson scheme is utilized. Thus the consistent linearization yields the corresponding total (algorithmic) tangent stiffness matrix defined by

$$
\begin{gathered}
{ }^{\text {tot }} \mathbf{K}:=\frac{\partial^{\text {tot }} \mathbf{R}}{\partial \mathbf{d}} \text { where, }{ }^{\text {tot }} \mathbf{K}=\left[\mathbf{K}^{I J}\right]+\left[{ }^{\text {tot }} \overline{\mathbf{K}}^{I J}\right] \text { with } \\
{ }^{\text {tot }} \overline{\mathbf{K}}^{I J}=\underbrace{\overline{\mathbf{K}}^{I J}}_{\text {interface in-plane }}+\underbrace{\overline{\mathbf{K}^{ \pm} J^{ \pm}}}_{\text {interface out-of-plane }} .
\end{gathered}
$$

Note that ${ }^{\text {tot }} \mathbf{K}$ is decomposed into contributions from the bulk $\mathbf{K}$ and the interface ${ }^{\text {tot }} \overline{\mathbf{K}}$. For a local damage model on the interface, the bulk, the elastic-damage interface and cohesive interface nodal stiffness sub-matrices $\mathbf{K}^{I J}, \overline{\mathbf{K}}^{I J}$ and $\overline{\mathbf{K}}^{I^{ \pm} J^{ \pm}}$are defined, respectively, by

$$
\begin{aligned}
& \mathbf{K}^{I J}=\frac{\partial \mathbf{R}^{I}}{\partial \boldsymbol{\varphi}^{J}}=\int_{\mathcal{B}_{0}} \operatorname{Grad} N^{I} \cdot \mathbb{A} \cdot \operatorname{Grad} N^{J} \mathrm{~d} V, \\
& \overline{\mathbf{K}}^{I J}=\frac{\partial \overline{\mathbf{R}}^{I}}{\partial \overline{\boldsymbol{\varphi}}^{J}}=\int_{\mathcal{I}_{0}} \overline{\operatorname{Grad}} \bar{N}^{I}: \overline{\mathbb{A}} \cdot \overline{\operatorname{Grad}} \bar{N}^{J} \mathrm{~d} A,
\end{aligned}
$$

$$
\begin{aligned}
\overline{\mathbf{K}}^{I^{ \pm} J^{ \pm}} & =\frac{\partial \overline{\mathbf{R}}^{I^{ \pm}}}{\partial \varphi^{J^{ \pm}}}=\mp \int_{\mathcal{I}_{0}} \frac{\partial \boldsymbol{T}}{\partial \varphi^{J^{ \pm}}} \overline{N^{I}} \mathrm{~d} A \\
& =\mp \pm \int_{\mathcal{I}_{0}} \mathcal{T} \bar{N}^{I} \bar{N}^{J} \mathrm{~d} A .
\end{aligned}
$$

where $\overline{\mathbb{A}}$ and $\mathcal{T}$ are calculated using Eqs. (30) and (31) and $\mathbb{A}=\partial \boldsymbol{P} / \partial \boldsymbol{F}$.

In the case of a non-local damage implementation, the elastic-damage interface nodal matrix Eq. (39) takes the form

$$
\begin{aligned}
\overline{\mathbf{K}}^{I J}= & \int_{\mathcal{I}_{0}} \overline{\operatorname{Grad}} \bar{N}^{I}\left(\overline{\boldsymbol{x}}_{\mathrm{r}}\right)-\left[\overline{\mathbb{A}}_{\mathrm{sect}}\left(\overline{\boldsymbol{x}}_{\mathrm{r}}\right)\right] \cdot \overline{\operatorname{Grad}} \bar{N}^{J}\left(\overline{\boldsymbol{x}}_{\mathrm{r}}\right) \mathrm{d} A_{\mathrm{r}} \\
& -\int_{\mathcal{I}_{0}}\left[1-\bar{D}_{\Varangle}\left(\overline{\boldsymbol{x}}_{\mathrm{r}}\right)\right] \overline{\operatorname{Grad}} \bar{N}^{I}\left(\overline{\boldsymbol{x}}_{\mathrm{r}}\right) \cdot \overline{\mathscr{D}}^{\prime}\left(\overline{\boldsymbol{x}}_{\mathrm{r}}\right) \overline{\boldsymbol{P}}_{0}\left(\overline{\boldsymbol{x}}_{\mathrm{r}}\right) \\
& \otimes\left[\int_{\mathcal{I}_{0}} \bar{\omega}\left(\overline{\boldsymbol{x}}_{\mathrm{r}}, \overline{\boldsymbol{x}}_{\mathrm{s}}\right) \frac{\partial \bar{F}_{\mathrm{loc}}\left(\overline{\boldsymbol{x}}_{\mathrm{s}}\right)}{\partial \overline{\boldsymbol{F}}\left(\overline{\boldsymbol{x}}_{\mathrm{s}}\right)} \cdot \overline{\operatorname{Grad}} \bar{N}^{J}\left(\overline{\boldsymbol{x}}_{\mathrm{s}}\right) \mathrm{d} A_{\mathrm{s}}\right] \mathrm{d} A_{\mathrm{r}},
\end{aligned}
$$

where $\overline{\mathscr{D}}^{\prime}$ is the derivative of $\overline{\mathscr{D}}$ with respect to its argument, $\overline{\mathscr{D}}^{\prime}=0$ in the case of unloading, $\mathrm{d} A_{\mathrm{S}}=\mathrm{d} A\left(\overline{\boldsymbol{x}}_{\mathrm{s}}\right)$ and $\mathrm{d} A_{\mathrm{r}}=$ $\mathrm{d} A\left(\bar{x}_{\mathrm{r}}\right)$. Note that the second term in Eq. (41) contributes to the interface stiffness matrix due to the non-locality of the interface damage model.

\section{Numerical examples}

The objective of this section is to study the role of a generalized mechanical interface (obeying tangential elasticity coupled to non-local damage and cohesive degradation) on the overall response of a body and to elucidate the theory presented in the previous sections. The influence of increasing specimen size on the overall response is also studied by fixing the bulk material parameters and increasing those of the interface. It is important to point out that the solution procedure is robust and shows the asymptotic quadratic rate of convergence associated with the Newton-Raphson scheme as expected from the consistently derived (algorithmic) stiffness matrices.

The material behavior in the bulk and on the tangential plane of the interface is characterized by Helmholtz energy functions. Table 3 gathers the effective (undamaged) constitutive relations in the bulk and on the generalized interface. The corresponding material parameters for the bulk and interface are given in Table 4. Note that some of the interface material parameters take different values than those appear- 
Table 3 Constitutive relations in the bulk and on the generalized interface in the material configuration

\begin{tabular}{|c|c|}
\hline \multicolumn{2}{|l|}{$\begin{array}{l}\Psi(\boldsymbol{F})=\frac{1}{2} \lambda \ln ^{2} J+\frac{1}{2} \mu[\boldsymbol{F}: \boldsymbol{F}-3-2 \ln J] \\
\boldsymbol{P}=\lambda \ln J \boldsymbol{F}^{-\mathrm{t}}+\mu\left[\boldsymbol{F}-\boldsymbol{F}^{-\mathrm{t}}\right] \\
\mathbb{A}=\lambda\left[\boldsymbol{F}^{-\mathrm{t}} \otimes \boldsymbol{F}^{-\mathrm{t}}+\ln J \mathbb{D}\right]+\mu[\mathbb{I}-\mathbb{D}]\end{array}$} \\
\hline In-plane & Out-of-plane \\
\hline $\bar{\Psi}_{0}(\overline{\boldsymbol{F}})=\frac{1}{2} \bar{\lambda} \ln ^{2} \bar{J}+\frac{1}{2} \bar{\mu}[\overline{\boldsymbol{F}}: \overline{\boldsymbol{F}}-2-2 \ln \bar{J}]$ & $\bar{\Psi}_{\nVdash}^{\sharp 0}=\exp (1) \sigma_{\mathrm{c}} \delta_{\mathrm{c}}\left[1-\left[1+|\llbracket \boldsymbol{\varphi} \rrbracket| / \delta_{\mathrm{c}}\right] \exp \left(-|\llbracket \boldsymbol{\varphi} \rrbracket| / \delta_{\mathrm{c}}\right)\right]$ \\
\hline$\overline{\boldsymbol{P}}_{0}=\bar{\lambda} \ln \bar{J} \overline{\boldsymbol{F}}^{-\mathrm{t}}+\bar{\mu}\left[\overline{\boldsymbol{F}}-\overline{\boldsymbol{F}}^{-\mathrm{t}}\right]$ & $\boldsymbol{T}_{0}=\exp (1) \sigma_{\mathrm{c}}|\llbracket \boldsymbol{\varphi} \rrbracket| \delta_{\mathrm{c}}^{-1} \exp \left(-|\llbracket \boldsymbol{\varphi} \rrbracket| / \delta_{\mathrm{c}}\right) \llbracket \boldsymbol{\varphi} \rrbracket /|\llbracket \boldsymbol{\varphi} \rrbracket|$ \\
\hline$\overline{\mathbb{A}}_{0}=\bar{\lambda}\left[\overline{\boldsymbol{F}}^{-\mathrm{t}} \otimes \overline{\boldsymbol{F}}^{-\mathrm{t}}+\ln \bar{J} \overline{\mathbb{D}}\right]+\bar{\mu}[\overline{\mathbb{I}}-\overline{\mathbb{D}}]$ & $\mathcal{T}_{0}=-\delta_{\mathrm{c}}^{-2} \exp (1) \sigma_{\mathrm{c}}|\llbracket \boldsymbol{\varphi} \rrbracket| \exp \left(-|\llbracket \boldsymbol{\varphi} \rrbracket| / \delta_{\mathrm{c}}\right) \frac{\llbracket \boldsymbol{\varphi} \rrbracket}{|\llbracket \boldsymbol{\varphi} \rrbracket|} \otimes \frac{\llbracket \boldsymbol{\varphi} \rrbracket}{|\llbracket \boldsymbol{\varphi} \rrbracket|}$ \\
\hline & $\begin{array}{l}+\exp (1) \sigma_{\mathrm{c}} \frac{1}{\delta_{\mathrm{c}}} \exp \left(-|\llbracket \varphi \rrbracket| / \delta_{\mathrm{c}}\right) \frac{\llbracket \boldsymbol{| \varphi} \rrbracket}{|\llbracket \varphi \rrbracket|} \otimes \frac{\llbracket \varphi \rrbracket}{|\llbracket \varphi \rrbracket|} \\
+\frac{1}{|\llbracket \varphi \rrbracket|} \exp (1) \sigma_{\mathrm{c}} \frac{|\llbracket \varphi \rrbracket|}{\delta_{\mathrm{c}}} \exp \left(-|\llbracket \varphi \rrbracket| / \delta_{\mathrm{c}}\right)\left[I-\frac{\llbracket \varphi \rrbracket}{|\llbracket \varphi \rrbracket|} \otimes \frac{\llbracket \varphi \rrbracket}{|\llbracket \varphi \rrbracket|}\right.\end{array}$ \\
\hline$\overline{\mathbb{I}}=\frac{\partial \overline{\boldsymbol{F}}}{\partial \overline{\boldsymbol{F}}}=\boldsymbol{i} \bar{\otimes} \overline{\boldsymbol{I}}$ & $\overline{\mathbb{D}}=\frac{\partial \overline{\boldsymbol{F}}^{-\mathrm{t}}}{\partial \overline{\boldsymbol{F}}}=-\overline{\boldsymbol{F}}^{-\mathrm{t}} \underline{\otimes}^{-1}+[\boldsymbol{i}-\overline{\boldsymbol{i}}] \bar{\otimes} \overline{\boldsymbol{F}}^{-1} \cdot \overline{\boldsymbol{F}}^{-\mathrm{t}}$ \\
\hline
\end{tabular}

Table 4 Material properties of the numerical examples

\begin{tabular}{lllll}
\hline & Bulk & & Interface \\
\hline Lamé constant & $\mu$ & $80193.8 \mathrm{~N} / \mathrm{mm}^{2}$ & $\bar{\mu}^{*}$ & $2 \times 80193.8 \mathrm{~N} / \mathrm{mm}$ \\
Lamé constant & $\lambda$ & $110743.5 \mathrm{~N} / \mathrm{mm}^{2}$ & $\bar{\lambda}^{*}$ & $2 \times 110743.5 \mathrm{~N} / \mathrm{mm}$ \\
& $E=\frac{\mu[3 \lambda+2 \mu]}{\mu+\lambda}$ & $206.9 \mathrm{~N} / \mathrm{mm}^{2}$ & $\bar{E}^{*}=4 \frac{\bar{\mu}[\bar{\lambda}+\bar{\mu}]}{2 \bar{\mu}+\bar{\lambda}}$ & $451.8 \mathrm{~N} / \mathrm{mm}$ \\
\hline & Only interface & & & $2000 \mathrm{~N} / \mathrm{mm}^{2}$ \\
& $\bar{F}_{0}$ & 0.0001 & $\sigma_{\mathrm{c}}$ & $0.2 \mathrm{~mm}$ \\
& $\bar{F}_{\mathrm{f}}$ & 0.1 & $\delta_{\mathrm{c}}$ & \\
\hline
\end{tabular}

* These variables take various values. Unless otherwise stated, the values appearing here are used for the numerical examples

ing in Table 4, which will be explicitly mentioned whenever necessary.

Consider the three-dimensional strip shown in Fig. 7. The strip is partitioned into two homogeneous domains by an interface. The width and the thickness of the strip are kept constant. A displacement of $0.5 \mathrm{~mm}$ is prescribed on the two opposite sides, resulting in a constant global loading of the strip. Under such conditions the resultant deformations are large; thus a finite deformation setting is required and implemented. The prescribed displacement is applied in 100 equal steps. The strip is discretized using 1600 trilinear hexahedral elements.

Figure 8 shows the evolution of the overall stress response measured in the bulk in the presence of a generalized interface at different interface elastic parameters. Note that interface elastic parameters vary while fixing the bulk Lamé parameters.

It should be pointed out that in the case of $\bar{\mu}=\bar{\lambda}=0$ the generalized interface is no longer elastically energetic and behaves like a classical cohesive interface (see Fig. 8a-d). Such behavior results in a stress concentration in the middle of the interface. The reason why the stress concentration occurs in the middle of the interface is that the highest value of the effective opening displacement occurs in that region. Consequently when $|\llbracket \varphi \rrbracket|$ reaches its critical value $\delta_{\mathrm{c}}$, traction reduction over the interface alleviates the intensity of the stress concentration.

Transitioning from a classical cohesive interface to a generalized interface dramatically changes the stress distribution in the domain (see Fig. 8e-p). Firstly such a change is clearly 


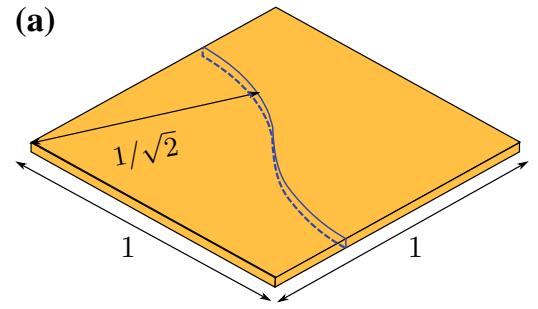

four side surfaces fixed in $y$ direction

top \& bottom surfaces fixed in $z$ direction

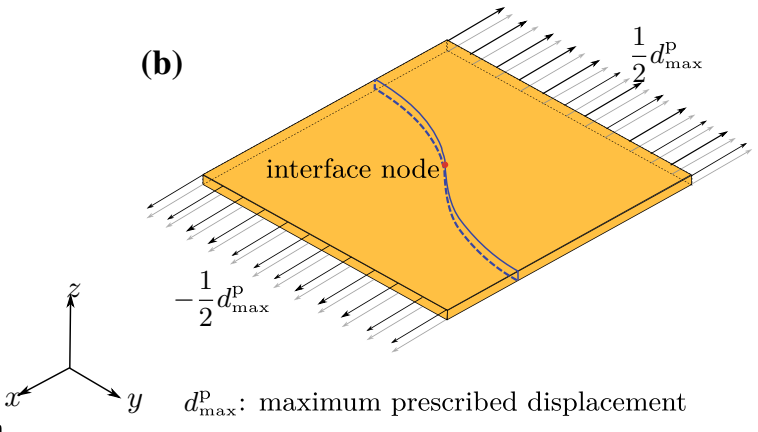

(b)

Fig. 7 Strip with curved interface: geometry a and applied boundary conditions $\mathbf{b}$. The maximum extension is $d_{\mathrm{max}}^{\mathrm{p}}=1 \mathrm{~mm}$. Dimensions are in $\mathrm{mm}$. The thickness is $0.05 \mathrm{~mm}$ increasing deformation

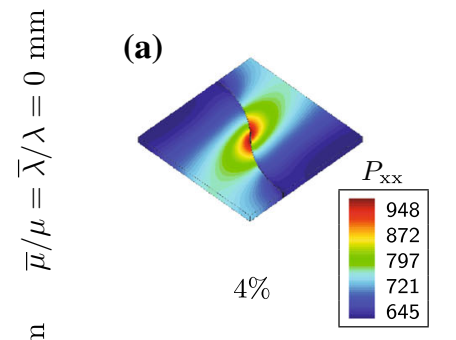

(b)

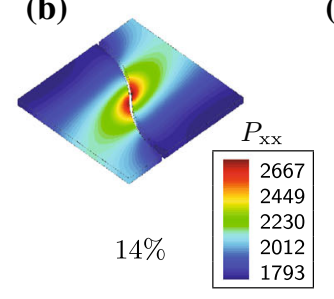

(e)

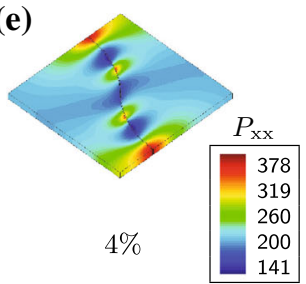

(i)

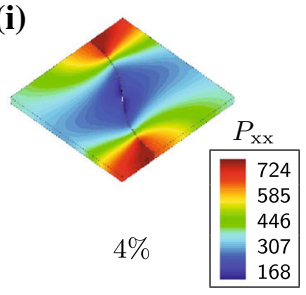

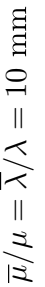

(f)

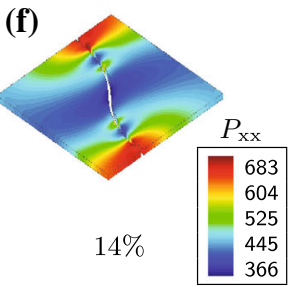

(j)

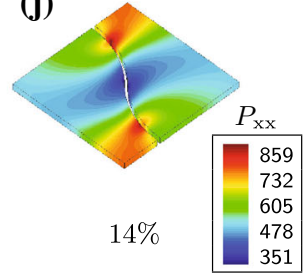

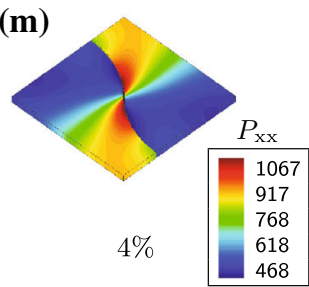

(n)

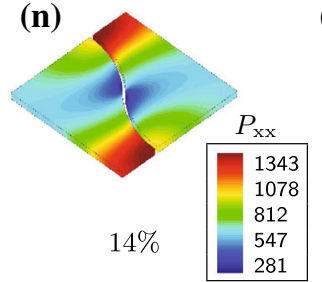

(c)

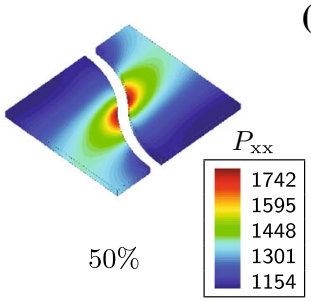

(g)

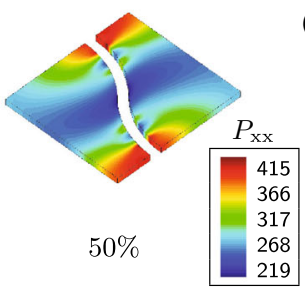

(k)

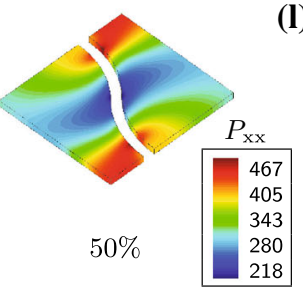

(d)

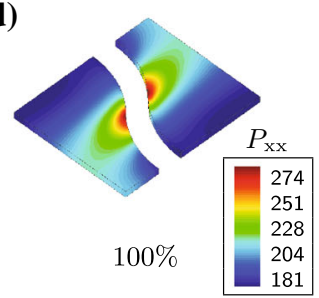

(h)

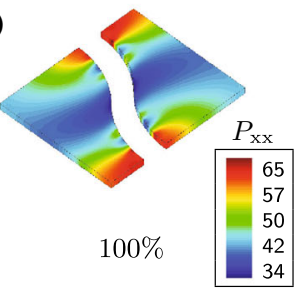

(l)

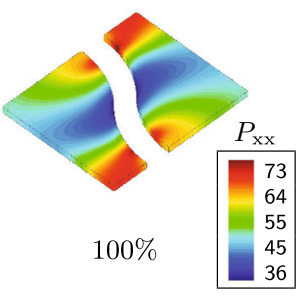

(o)

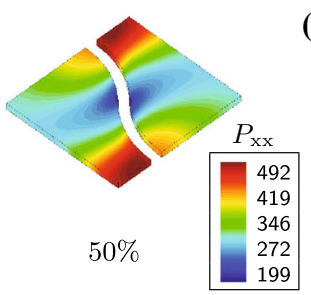

(p)

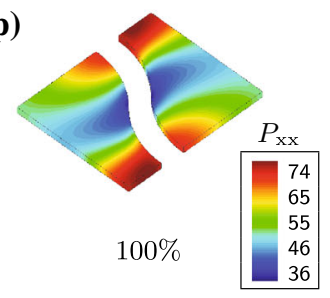

Fig. 8 Bulk stress distribution in the presence of the generalized interface subject to in-plane degradation at $4,14,50$, and $100 \%$ of the final deformation $d_{\max }^{\mathrm{p}}$. The results a-d correspond to the bulk containing a cohesive interface without surface energy $(\bar{\mu} / \mu=$ $\bar{\lambda} / \lambda=0 \mathrm{~mm})$, respectively. The results $\mathbf{e}-\mathbf{h}$ correspond to the bulk containing a generalized interface with $\bar{\mu} / \mu=\bar{\lambda} / \lambda=0.0001$ $\mathrm{mm}$, respectively. The results $\mathbf{i}-\mathbf{l}$ correspond to the bulk containing a generalized interface with $\bar{\mu} / \mu=\bar{\lambda} / \lambda=2 \mathrm{~mm}$, respectively. The results $\mathbf{m}-\mathbf{p}$ correspond to the bulk containing a generalized interface with $\bar{\mu} / \mu=\bar{\lambda} / \lambda=10 \mathrm{~mm}$, respectively. The stress depicted is the $\mathrm{xx}$-component of the Piola stress tensor in unit of $\mathrm{N} / \mathrm{mm}^{2}$. (Color figure online) 
observed in the shape, location and magnitude of the stress concentrations due to the tangential degradation of the interface. In all the cases depicted in Fig. 8e-p compared to Fig. 8a-d the two distinct zones of concentrated stress are further apart along the interface. Secondly a comparison of the stress distribution of all the cases at $100 \%$ of the prescribed deformation (compare Fig. $8 d$ with $h, 1$ and p) reveals an overall drop in stress. This is another consequence of a mechanically weakened (damaged) interface in its tangential plane in addition to its decohesion across the interface. Thirdly having compared Fig. 8i-1 with Fig. 8m-p respectively, one can deduce that the more evolved the total damage $D_{\text {tot }}$, the more similar the stress distributions. This observation stems from the degradation of interface material with the evolution of damage where at high deformation levels the interface no longer bears any load. It is also of interest to note that as one moves from a classical cohesive interface to a generalized (cohesive and energetic) interface a drop in stress is observed (see for instance the first column in Fig. 8). This is due to the fact that for a purely classical cohesive interface, the traction across the interface assumes the highest value (see Fig. 11c), inducing large stress especially in the middle of the interface. As the interface becomes energetic, the evolution of tangential damage $\bar{D}_{\|}$dramatically decreases the traction value (see Figs. 11c and 12a) and thus causes the drop in the stress measured.

The bulk stress evolution is shown in Fig. 11a. One can observe two major drops in stress associated with

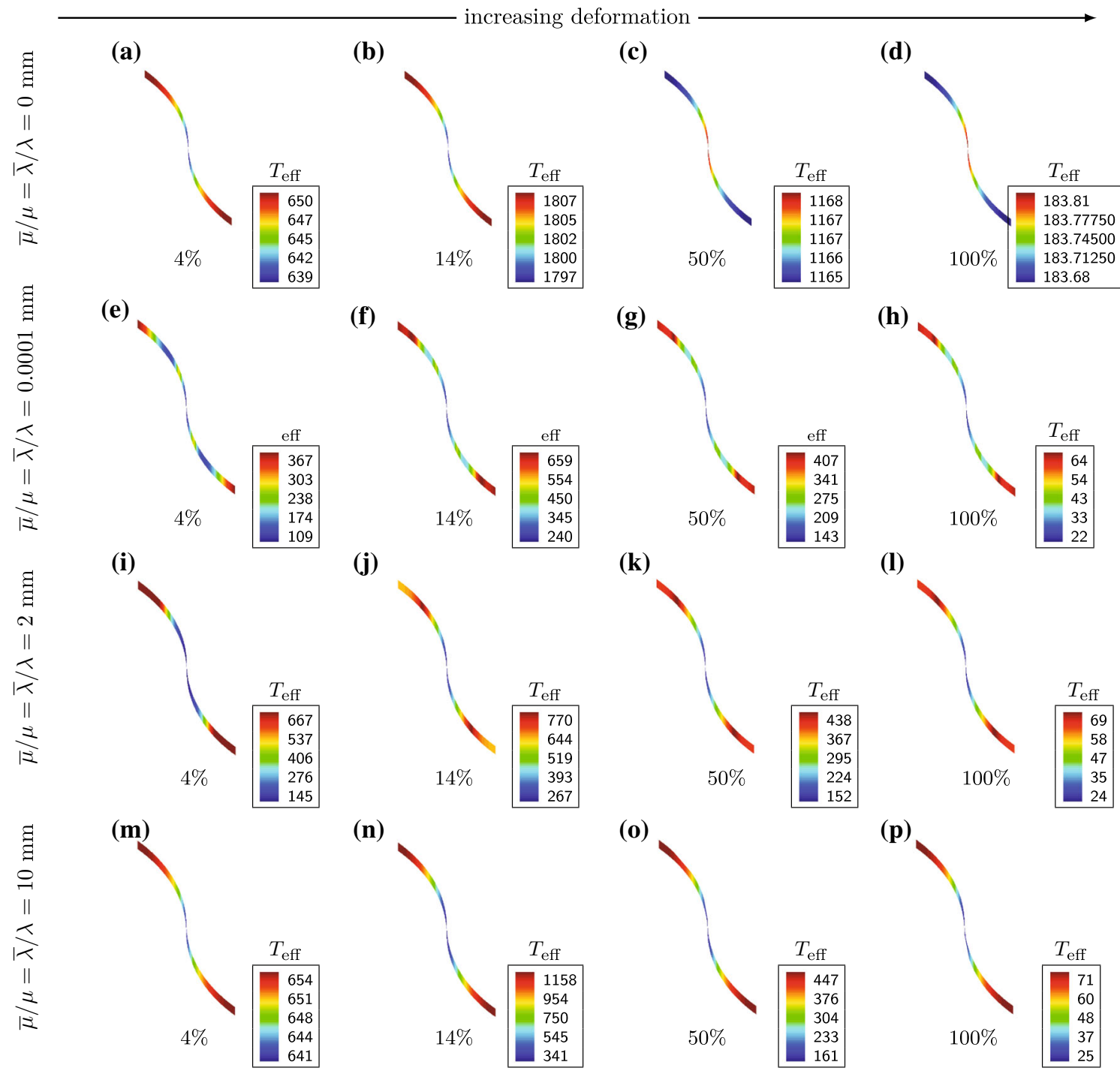

Fig. 9 Interface traction distribution with and without tangential life at $4,14,50$, and $100 \%$ of the final deformation $d_{\max }^{\mathrm{p}}$. The results $\mathbf{a}-\mathbf{d}$ correspond to an interface without surface energy $(\bar{\mu} / \mu=\bar{\lambda} / \lambda=0$ $\mathrm{mm})$, respectively. The results $\mathbf{e}-\mathbf{h}$ correspond to a generalized interface with $\bar{\mu} / \mu=\bar{\lambda} / \lambda=0.0001 \mathrm{~mm}$, respectively. The results $\mathbf{i}-\mathbf{l}$ correspond to a generalized interface with $\bar{\mu} / \mu=\bar{\lambda} / \lambda=2 \mathrm{~mm}$, respectively. The results $\mathbf{m}-\mathbf{p}$ correspond to a generalized interface with $\bar{\mu} / \mu=\bar{\lambda} / \lambda=10 \mathrm{~mm}$, respectively. The traction unit is $\mathrm{N} / \mathrm{mm}^{2}$. (Color figure online) 


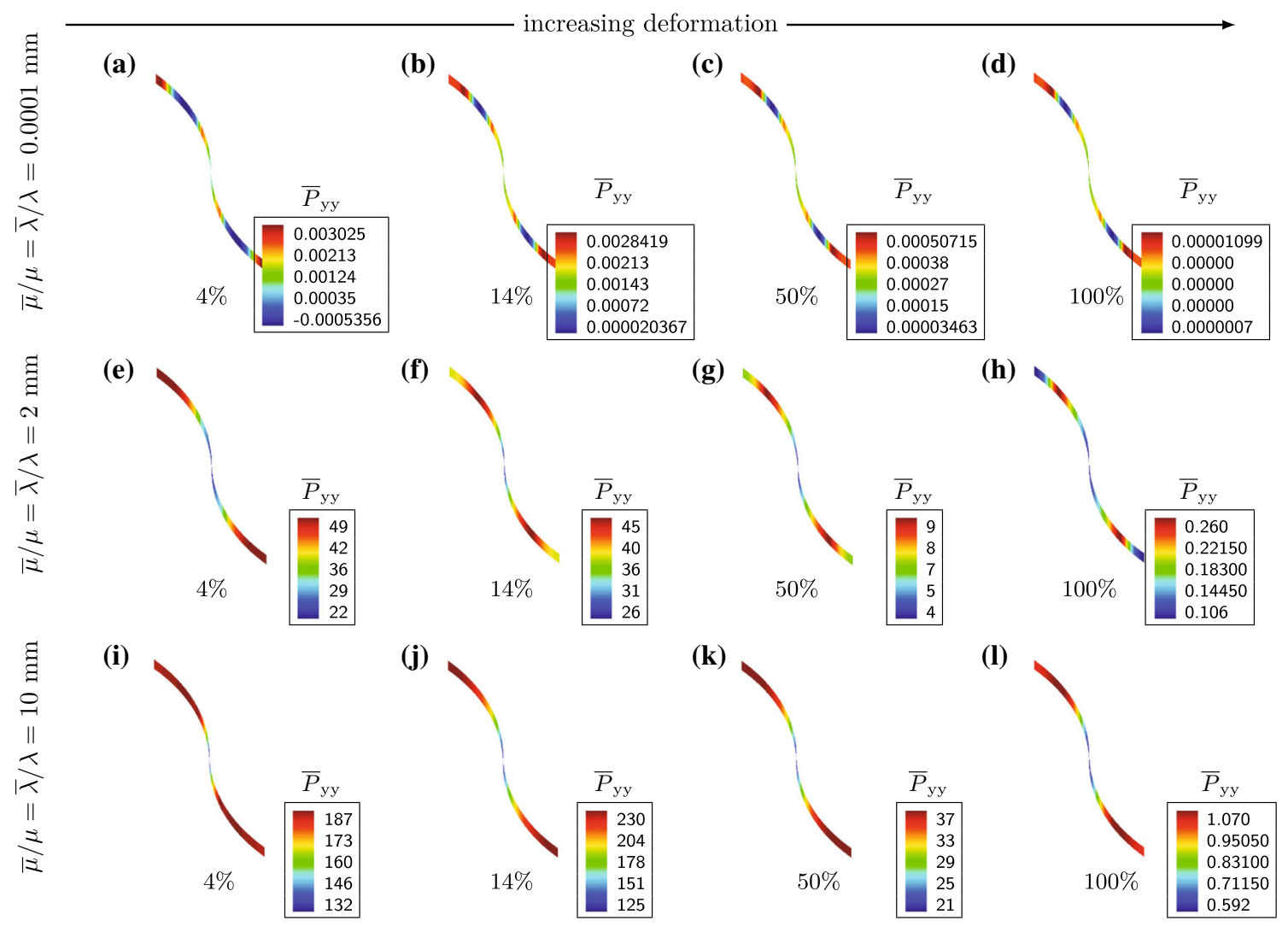

Fig. 10 Interface stress distribution at $4,14,50$, and $100 \%$ of the final deformation $d_{\max }^{\mathrm{p}}$. The results a-d correspond to interface with $\bar{\mu} / \mu=\bar{\lambda} / \lambda=0.0001 \mathrm{~mm}$, respectively. The results $\mathbf{e}-\mathbf{h}$ correspond to the generalized interface with $\bar{\mu} / \mu=\bar{\lambda} / \lambda=2 \mathrm{~mm}$, respec-

the onset of tangential and cohesive damage, respectively. Note that both tangential and cohesive damage variables $\bar{D}_{\|}$and $\bar{D}_{\Varangle}$ contribute to the total damage $\bar{D}_{\text {tot }}$. Therefore any increase in the cohesive damage adds to the localized distortion.

The traction response of the generalized interface with and without tangential damage is illustrated in Fig. 9a-d and e$\mathrm{p}$, respectively. With $\bar{\mu} / \mu=\bar{\lambda} / \lambda=0$ presenting a classical cohesive interface, the traction first reaches a critical value $\sigma_{\mathrm{c}}$ and in the following decays exponentially. Such behavior is first observed in the middle of the interface possessing the highest value of the effective opening. On the contrary, for all the generalized interfaces $(\bar{\mu} / \mu=\bar{\lambda} / \lambda \neq 0)$ the tractions always stay beneath the critical value $\delta_{\mathrm{c}}=2000 \mathrm{~N} / \mathrm{mm}^{2}$ which is caused by the tangential degradation of the interface. Considering the minimum values of the tractions reveals that the stiffer interfaces have the larger traction drops at $50 \%$ of final deformation (see Fig. 9g, k and o for example). This observation is also illustrated in Fig. 11c.

The interface stress profiles are shown in Fig. 10. We observe that with increasing the interface elasticity the location of minimum stress and the deformation level at which tively. The results $\mathbf{i}-\mathbf{l}$ correspond to the generalized interface with $\bar{\mu} / \mu=\bar{\lambda} / \lambda=10 \mathrm{~mm}$, respectively. The stress depicted is the yycomponent of the first Piola stress tensor in unit of N/mm. (Color figure online)

the stress drop occurs are different. For instance the stress drop for the case $\bar{\mu} / \mu=\bar{\lambda} / \lambda=2$ is seen at $14 \%$ of total stretch, yet such drop in the case of $\bar{\mu} / \mu=\bar{\lambda} / \lambda=10$ takes place at $50 \%$ of final deformation.

In addition the evolution of interface stress depicted in Fig. $11 \mathrm{~b}$ consists of a gradual reduction of the stress with the onset of $\bar{D}_{\|}$, a sudden drop of the stress with the onset of $\bar{D}_{\nVdash}$ and finally an exponential decay of the stress with the evolution of both $\bar{D}_{\|}$and $\bar{D}_{\Varangle}$.

The evolution of the tangential, cohesive and total damage profiles together with the equivalent distortion are demonstrated in Fig. 12a-d, respectively. It is observed that the higher the interface energy the more delayed is the onset of the tangential damage. Furthermore, in general the more elastic interface undergoes smaller tangential deformation and thus in-plane degradation (see Fig. 12a, d). It is also of interest to note that with the onset of cohesive damage a sudden increase in equivalent distortion of all the interfaces is observed which is directly translated into higher tangential and total degradation of the interface. Nonetheless, the more energetic interfaces depict higher sensitivity to the initiation of interface cohesive damage. Finally the saw-tooth behavior 
(a)

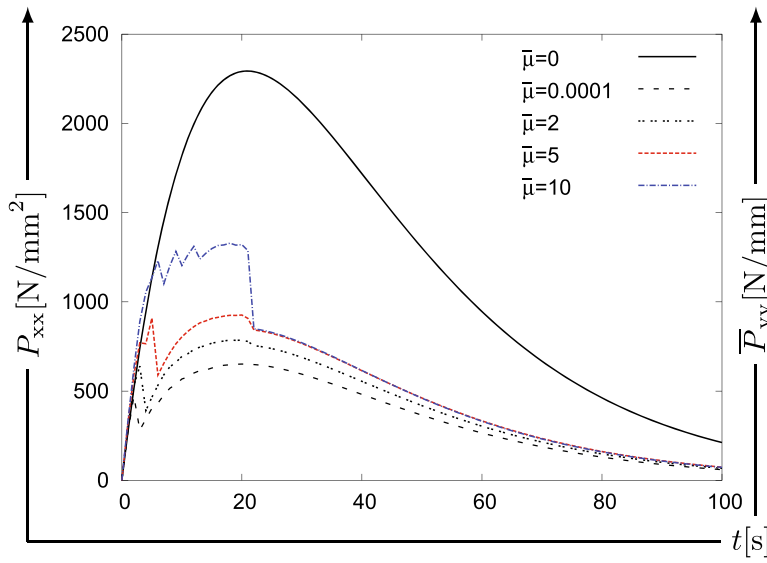

(b)

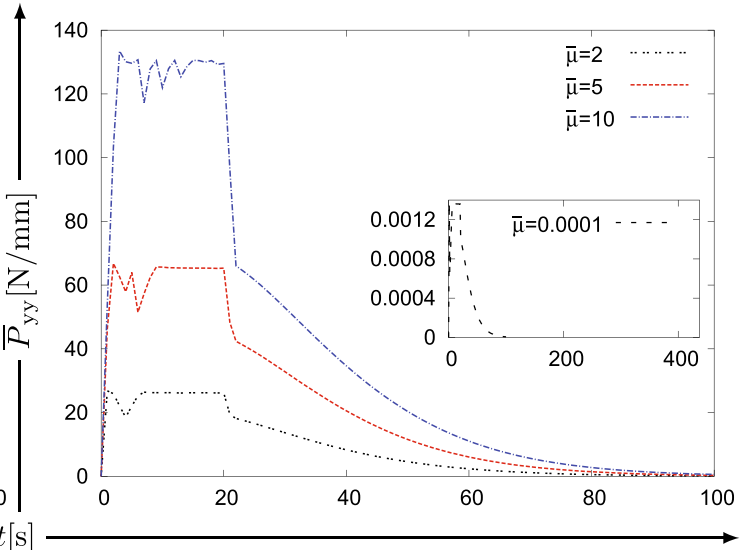

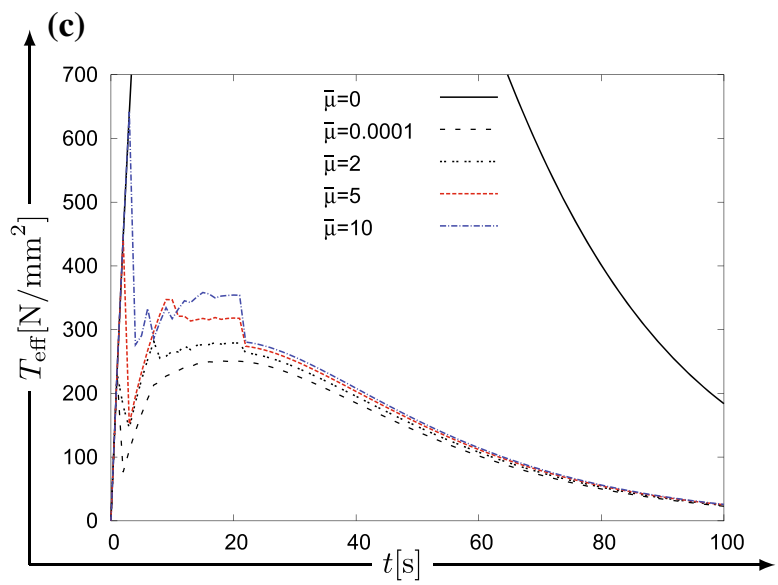

Fig. 11 Evolution of bulk Piola stress (a), interface Piola stress (b) and interface traction (c). The stresses and traction are measured on the node in the middle of the domain (see Fig. 7b) for the interfaces with $\bar{\mu} / \mu=\bar{\lambda} / \lambda=0,0.0001,2,5$ and $10 \mathrm{~mm}$. (Color figure online)

observed in Fig. 11 is due to the existence of the bifurcation point in the tangential stress response as illustrated in Fig. $6 \mathrm{~b}$. Moreover one needs to take into account the effect of tangential damage on the cohesive traction: as we increase the loading, the tangential damage grows and consequently the cohesive traction is reduced, which removes loading from the interface, resulting in a local unloading on the interface. This response is observed until the onset of cohesive damage occurs.

\section{Summary and conclusion}

A three-dimensional finite element framework for continua containing geometrically non-linear generalized interfaces is presented. The tangential elastic response of the interface is captured by endowing it with its own mechanically energetic structure. The interface in-plane degradation is modeled using an integral-type non-local isotropic damage approach. In addition, an irreversible large-deformation cohesive zone model is used to capture the material decohesion across the interface. The material degradation along and across the interface are coupled through the tangential and cohesive damage variables. The corresponding weak forms of the balance equation including the contributions from the interface are given. The balance equations are fully discretized using the finite element method. A material model for the interface Helmholtz energy is then introduced. An exponential traction-separation law is selected to describe the interface cohesive constitutive relation. The exact consistent stiffness matrices in the bulk and on the interface are derived.

A series of three-dimensional numerical examples serves to elucidate the role of the novel generalized interface model on the overall response of the body. A comparison of the results obtained from classical and novel (current work) interface models reveals that the location, shape and intensity of stress concentrations are dramatically affected. In addition, the stress response of the body is at lower levels due to the tangential and cohesive interface material damage. It is also shown that tangential damage dramatically increases with the onset of the cohesive damage. 
(a)

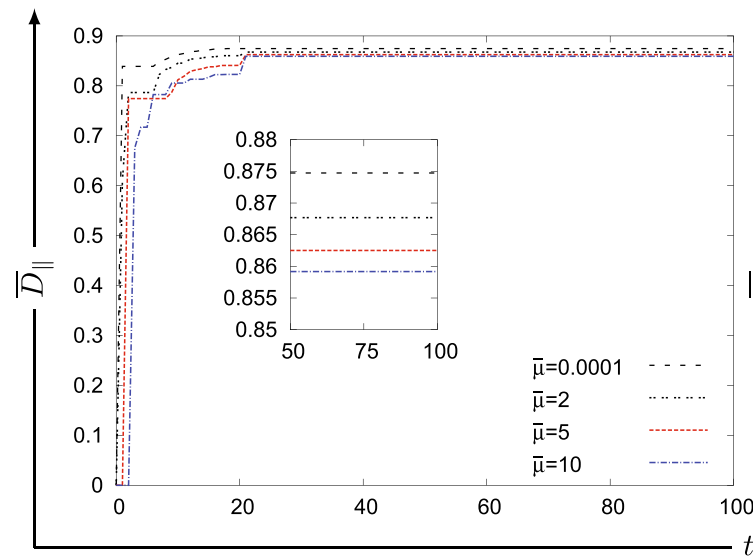

(b)

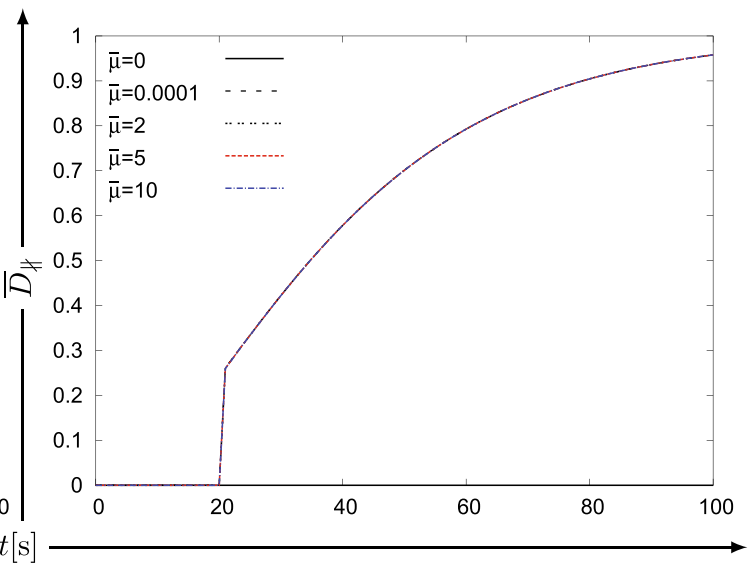

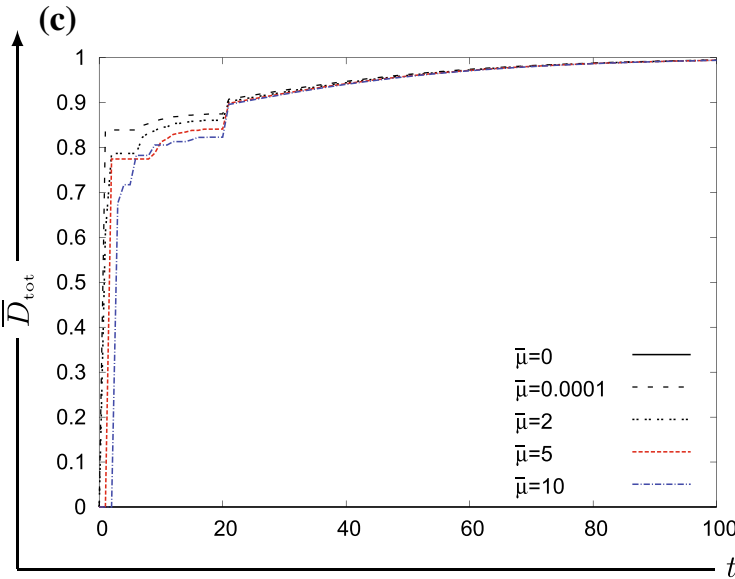

Fig. 12 Evolution of tangential damage variable (a), cohesive damage variable (b), total damage variable (c) and non-local equivalent distortion (d). The damage variables and the non-local equivalent distortion

It is straightforward to employ more sophisticated damage mechanisms such as anisotropic damage models. Moreover, an investigation of the influences of the current interface model on the thermo-mechanical response of the body is of great importance. These extensions shall be discussed in later contributions.

Acknowledgements This research is performed as part of the Energie Campus Nuremberg and supported by funding through the "Bavaria on the Move" initiative of the state of Bavaria. The authors also gratefully acknowledge the support by the Cluster of Excellence "Engineering of Advanced Materials".

\section{Appendix: Some mathematical relations and derivations}

In this section we derive the weak form of the balance of forces. Some useful identities and relations used in the derivations are also given without proof.

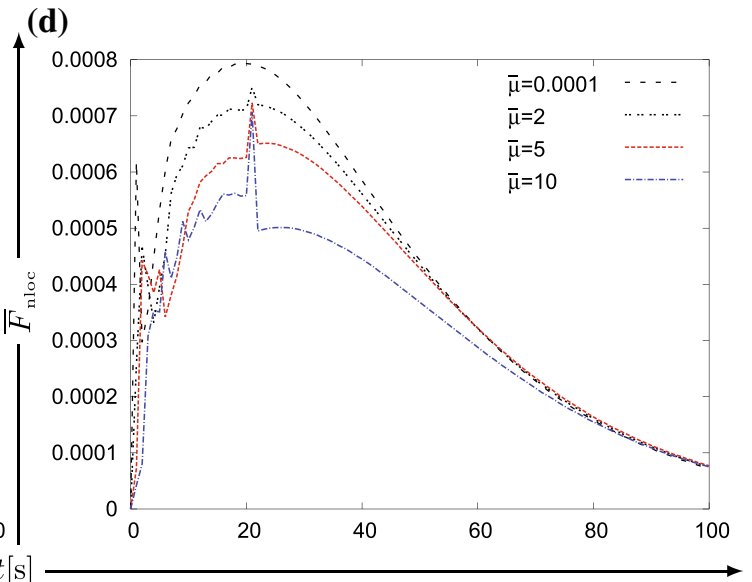

are measured on the node in the middle of the domain (see Fig. 7b) for the interfaces with $\bar{\mu} / \mu=\bar{\lambda} / \lambda=0,0.0001,2,5$ and $10 \mathrm{~mm}$. (Color figure online)

\section{Extended divergence theorem}

The extended forms of the divergence theorem in the material configuration for a bulk tensor field $\{\bullet\}$ and a tensorial quantity on the interface $\{\overline{\boldsymbol{\theta}}\}$ are

$$
\begin{aligned}
\int_{\mathcal{B}_{0}} \operatorname{Div}\{\bullet\} \mathrm{d} V= & \int_{\partial \mathcal{B}_{0}}\{\bullet\} \cdot N \mathrm{~d} A-\int_{\mathcal{I}_{0}} \llbracket \bullet \cdot \overline{\boldsymbol{N}} \mathrm{d} A \\
\int_{\mathcal{I}_{0}} \overline{\operatorname{Div}}\{\overline{\boldsymbol{\bullet}}\} \mathrm{d} A= & \int_{\partial \mathcal{I}_{0} \backslash \partial \mathcal{I}_{0}^{\mathrm{N}}}\{\bar{\bullet}\} \cdot \widetilde{\boldsymbol{N}} \mathrm{d} L+\int_{\partial \mathcal{I}_{0}^{\mathrm{N}}}\{\overline{\boldsymbol{\bullet}}\} \cdot \widetilde{\boldsymbol{N}} \mathrm{d} L \\
& -\int_{\mathcal{I}_{0}} \bar{C}\{\bar{\bullet}\} \cdot \overline{\boldsymbol{N}} \mathrm{d} A
\end{aligned}
$$

where the curvature of the interface is denoted by $\bar{C}$. Note that $\partial \mathcal{I}_{0}^{\mathrm{N}}$ is the portion of the interface boundary that intersects with the bulk's boundary, thus $\partial \mathcal{I}_{0} \backslash \partial \mathcal{I}_{0}^{\mathrm{N}} \cap \partial \mathcal{B}_{0}=\emptyset$. 


\section{Balance of forces and moments on the interface}

The global form of the balance of forces both in the bulk and on the interface is given as [see [45], for further details concerning thermomechanical solids with surface energy only]

$$
\begin{gathered}
\int_{\mathcal{B}_{0}} \boldsymbol{b}^{\mathrm{p}} \mathrm{d} V+\int_{\mathcal{I}_{0}} \overline{\boldsymbol{b}}^{\mathrm{p}} \mathrm{d} A+\int_{\partial \mathcal{B}_{0}^{\mathrm{N}}} \widehat{\boldsymbol{b}}_{\mathrm{N}}^{\mathrm{p}} \mathrm{d} A+\int_{\partial \mathcal{I}_{0}^{\mathrm{N}}} \widetilde{\boldsymbol{b}}_{\mathrm{N}}^{\mathrm{p}} \mathrm{d} L \\
+\int_{\partial \mathcal{B}_{0} \backslash \partial \mathcal{B}_{0}^{\mathrm{N}}} \boldsymbol{P} \cdot \boldsymbol{N} \mathrm{d} A+\int_{\partial \mathcal{I}_{0} \backslash \partial \mathcal{I}_{0}^{\mathrm{N}}} \overline{\boldsymbol{P}} \cdot \widetilde{\boldsymbol{N}} \mathrm{d} L=\mathbf{0} .
\end{gathered}
$$

Taking the limit $\mathcal{B}_{0} \rightarrow \emptyset$, and consequently $\partial \mathcal{B}_{0}=\mathcal{I}_{0}$, with $\boldsymbol{N}=\overline{\boldsymbol{N}}$ on $\mathcal{I}_{0}^{+}, \boldsymbol{N}=-\overline{\boldsymbol{N}}$ on $\mathcal{I}_{0}^{-}, \partial \mathcal{I}_{0}^{\mathrm{N}}=\emptyset, \partial \mathcal{B}_{0}^{\mathrm{N}}=\varnothing$, and taking into account the extended forms of the divergence theorem (42) and (43), one obtains the local balance of forces on the interface as

$$
\begin{gathered}
\int_{\partial \mathcal{I}_{0}} \overline{\boldsymbol{P}} \cdot \tilde{\boldsymbol{N}} \mathrm{d} L+\int_{\mathcal{I}_{0}} \boldsymbol{P} \cdot \boldsymbol{N} \mathrm{d} A+\int_{\mathcal{I}_{0}} \overline{\boldsymbol{b}}^{\mathrm{p}} \mathrm{d} A=\mathbf{0} \Longrightarrow \\
\int_{\mathcal{I}_{0}} \overline{\operatorname{Div}} \overline{\boldsymbol{P}}+\llbracket \boldsymbol{P} \rrbracket \cdot \overline{\boldsymbol{N}}+\overline{\boldsymbol{b}}^{\mathrm{p}} \mathrm{d} A=\mathbf{0} .
\end{gathered}
$$

From arbitrariness of $\mathcal{B}_{0}$ and thus $\mathcal{I}_{0}$, the balance of force on the interface listed in Table 2 then follows. In the case that the interface is not energetic i.e. $\overline{\boldsymbol{P}}=\mathbf{0}$, and in the absence of interface body force $\left(\overline{\boldsymbol{b}}^{\mathrm{p}}=\mathbf{0}\right)$, the classical traction continuity condition is recovered.

The global form of balance of moment in the absence of body forces reads

$\int_{\partial \mathcal{B}_{0}} \boldsymbol{r} \times[\boldsymbol{P} \cdot \boldsymbol{N}] \mathrm{d} A+\int_{\partial \mathcal{I}_{0}} \overline{\boldsymbol{r}} \times[\overline{\boldsymbol{P}} \cdot \widetilde{\boldsymbol{N}}] \mathrm{d} L=\mathbf{0}$,

where $\boldsymbol{r}$ and $\overline{\boldsymbol{r}}$ are the bulk and interface position vectors. Now by using the extended divergence theorem and the relations

$$
\begin{gathered}
\int_{\partial \mathcal{B}_{0}} \boldsymbol{r} \times \boldsymbol{P} \cdot \boldsymbol{N} \mathrm{d} A=\int_{\mathcal{B}_{0}} \boldsymbol{r} \times \operatorname{Div} \boldsymbol{P}+\boldsymbol{\varepsilon}: \boldsymbol{F} \cdot \boldsymbol{P}^{\mathrm{t}} \mathrm{d} V \text { and } \\
\int_{\partial \mathcal{I}_{0}} \overline{\boldsymbol{r}} \times \overline{\boldsymbol{P}} \cdot \overline{\boldsymbol{N}} \mathrm{d} L=\int_{\mathcal{I}_{0}} \overline{\boldsymbol{r}} \times \overline{\operatorname{Div} \boldsymbol{P}}+\boldsymbol{\varepsilon}: \overline{\boldsymbol{F}} \cdot \overline{\boldsymbol{P}}^{\mathrm{t}} \mathrm{d} A
\end{gathered}
$$

one obtains

$$
\begin{gathered}
\int_{\mathcal{B}_{0}} \boldsymbol{r} \times \operatorname{Div} \boldsymbol{P}+\boldsymbol{\varepsilon}: \boldsymbol{F}^{\mathrm{t}} \cdot \boldsymbol{P} \mathrm{d} V+\int_{\mathcal{I}_{0}} \boldsymbol{r} \times \llbracket \boldsymbol{P} \rrbracket \cdot \overline{\boldsymbol{N}} \mathrm{d} A \\
\quad+\int_{\mathcal{I}_{0}} \overline{\boldsymbol{r}} \times \overline{\operatorname{Div} \boldsymbol{P}}+\boldsymbol{\varepsilon}: \overline{\boldsymbol{F}} \cdot \overline{\boldsymbol{P}}^{\mathrm{t}} \mathrm{d} A=\mathbf{0} .
\end{gathered}
$$

By taking the limit $\mathcal{B}_{0} \rightarrow \emptyset$, noting that $\mathcal{I}_{0}^{+}=\mathcal{I}_{0}^{-}=\mathcal{I}_{0}, \overline{\boldsymbol{r}}=$ $\{\{\boldsymbol{r}\}$, and using the balance of forces on the interface, listed in Table 2, we find

$$
\begin{array}{r}
\int_{\mathcal{I}_{0}^{+}} \boldsymbol{r}^{+} \times \boldsymbol{P}^{+} \cdot \overline{\boldsymbol{N}} \mathrm{d} A-\int_{\mathcal{I}_{0}^{-}} \boldsymbol{r}^{-} \times \boldsymbol{P}^{-} \cdot \overline{\boldsymbol{N}} \mathrm{d} A \\
+\int_{\mathcal{I}_{0}}-\left\{\{\boldsymbol{r}\} \times \llbracket \boldsymbol{P} \rrbracket \cdot \overline{\boldsymbol{N}}+\boldsymbol{\varepsilon}: \overline{\boldsymbol{F}} \cdot \overline{\boldsymbol{P}}^{\mathrm{t}} \mathrm{d} A=\mathbf{0},\right.
\end{array}
$$

which simplifies to

$$
\begin{aligned}
& \int_{\mathcal{I}_{0}} \llbracket \boldsymbol{r} \rrbracket \times\left\{\{\boldsymbol{P}\} \cdot \overline{\boldsymbol{N}} \mathrm{d} A+\int_{\mathcal{I}_{0}} \boldsymbol{\varepsilon}: \overline{\boldsymbol{F}} \cdot \overline{\boldsymbol{P}}^{\mathrm{t}} \mathrm{d} A=\mathbf{0} \Longrightarrow\right. \\
& \llbracket \boldsymbol{r} \rrbracket \times \underbrace{\{\boldsymbol{P}\}] \cdot \overline{\boldsymbol{N}}}_{\boldsymbol{T}}+\boldsymbol{\varepsilon}: \overline{\boldsymbol{F}} \cdot \overline{\boldsymbol{P}}^{\mathrm{t}}=\mathbf{0} .
\end{aligned}
$$

Now by noting $\llbracket \boldsymbol{r} \rrbracket \times\{\{\boldsymbol{P}\} \cdot \overline{\boldsymbol{N}}=\boldsymbol{\varepsilon}:[\{\boldsymbol{P}\} \cdot \overline{\boldsymbol{N}} \otimes \llbracket \boldsymbol{r} \rrbracket]$ we have

$$
\begin{aligned}
& \varepsilon:\left[\{\boldsymbol{P}\} \cdot \overline{\boldsymbol{N}} \otimes \llbracket \boldsymbol{r} \rrbracket+\overline{\boldsymbol{F}} \cdot \overline{\boldsymbol{P}}^{\mathrm{t}}\right]=\mathbf{0} \Longrightarrow \\
& \quad\left[\{[\boldsymbol{P}\}] \cdot \overline{\boldsymbol{N}} \otimes \llbracket \boldsymbol{r} \rrbracket+\overline{\boldsymbol{F}} \cdot \overline{\boldsymbol{P}}^{\mathrm{t}}\right]=\left[\left\{\{\boldsymbol{P}\} \cdot \overline{\boldsymbol{N}} \otimes \llbracket \boldsymbol{r} \rrbracket+\overline{\boldsymbol{F}} \cdot \overline{\boldsymbol{P}}^{\mathrm{t}}\right]^{\mathrm{t}} .\right.
\end{aligned}
$$

Note that since in this work the cohesive traction $\boldsymbol{T}$ is colinear with the displacement jump vector $\llbracket \boldsymbol{r} \rrbracket$, the balance of moments on the interface becomes $\boldsymbol{\varepsilon}: \overline{\boldsymbol{F}} \cdot \overline{\boldsymbol{P}}^{\mathrm{t}}=\mathbf{0}$, thus $\overline{\boldsymbol{P}} \cdot \overline{\boldsymbol{F}}^{\mathrm{t}}=\overline{\boldsymbol{F}} \cdot \overline{\boldsymbol{P}}^{\mathrm{t}}$.

\section{Weak form of the balance of forces}

The localized balance equations in the bulk and on the interface, given in Table 2 are tested from the left with vector valued functions $\delta \boldsymbol{\varphi}$ and $\delta \overline{\boldsymbol{\varphi}}$, respectively as follows

$$
\begin{aligned}
& \int_{\mathcal{B}_{0}} \delta \boldsymbol{\varphi} \cdot\left[\operatorname{Div} \boldsymbol{P}+\boldsymbol{b}^{\mathrm{p}}\right] \mathrm{d} V \\
& \quad+\int_{\mathcal{I}_{0}} \delta \overline{\boldsymbol{\varphi}} \cdot\left[\overline{\operatorname{Div}} \overline{\boldsymbol{P}}+\overline{\boldsymbol{b}}^{\mathrm{p}}+\llbracket \boldsymbol{P} \rrbracket \cdot \overline{\boldsymbol{N}}\right] \mathrm{d} A=0 .
\end{aligned}
$$

which can be alternatively written as

$$
\begin{gathered}
\int_{\mathcal{B}_{0}}-\boldsymbol{P}: \operatorname{Grad} \delta \boldsymbol{\varphi}+\operatorname{Div}(\delta \boldsymbol{\varphi} \cdot \boldsymbol{P})+\delta \boldsymbol{\varphi} \cdot \boldsymbol{b}^{\mathrm{p}} \mathrm{d} V \\
+\int_{\mathcal{I}_{0}}-\overline{\boldsymbol{P}}: \overline{\operatorname{Grad}} \delta \overline{\boldsymbol{\varphi}}+\overline{\operatorname{Div}}(\delta \overline{\boldsymbol{\varphi}} \cdot \overline{\boldsymbol{P}}) \mathrm{d} A \\
+\int_{\mathcal{I}_{0}} \delta \overline{\boldsymbol{\varphi}} \cdot \overline{\boldsymbol{b}}^{\mathrm{p}}+\delta \overline{\boldsymbol{\varphi}} \cdot[\llbracket \boldsymbol{P} \rrbracket \cdot \overline{\boldsymbol{N}}] \mathrm{d} A=0,
\end{gathered}
$$

and using the extended forms of divergence theorem (42) and (43), for various parts of the body results in

$$
\begin{gathered}
\int_{\mathcal{B}_{0}} \boldsymbol{P}: \operatorname{Grad} \delta \varphi \mathrm{d} V-\int_{\partial_{\mathcal{B}_{0}^{N}}} \delta \boldsymbol{\varphi} \cdot[\boldsymbol{P} \cdot \boldsymbol{N}] \mathrm{d} A \\
+\int_{\mathcal{I}_{0}} \llbracket \delta \boldsymbol{\varphi} \cdot \boldsymbol{P} \rrbracket \cdot \overline{\boldsymbol{N}} \mathrm{d} A-\int_{\mathcal{B}_{0}} \delta \boldsymbol{\varphi} \cdot \boldsymbol{b}^{\mathrm{p}} \mathrm{d} V
\end{gathered}
$$




$$
\begin{aligned}
& +\int_{\mathcal{I}_{0}} \overline{\boldsymbol{P}}: \overline{\operatorname{Grad}} \delta \overline{\boldsymbol{\varphi}} \mathrm{d} A-\int_{\partial \mathcal{I}_{0}^{\mathrm{N}}} \delta \overline{\boldsymbol{\varphi}} \cdot[\overline{\boldsymbol{P}} \cdot \widetilde{\boldsymbol{N}}] \mathrm{d} L \\
& -\int_{\mathcal{I}_{0}} \delta \overline{\boldsymbol{\varphi}} \cdot \overline{\boldsymbol{b}}^{\mathrm{p}}+\delta \overline{\boldsymbol{\varphi}} \cdot[\llbracket \boldsymbol{P} \rrbracket \cdot \overline{\boldsymbol{N}}] \mathrm{d} A=0 .
\end{aligned}
$$

On the Neumann boundaries of the bulk and interface, $\boldsymbol{P} \cdot \boldsymbol{N}=\widehat{\boldsymbol{b}}_{\mathrm{N}}^{\mathrm{p}}$ and $\overline{\boldsymbol{P}} \cdot \widetilde{\boldsymbol{N}}=\widetilde{\boldsymbol{b}}_{\mathrm{N}}^{\mathrm{p}}$, respectively. Noting $\llbracket \delta \boldsymbol{\varphi} \cdot \boldsymbol{P} \rrbracket=$ $\llbracket \delta \varphi \rrbracket \cdot\{\{\boldsymbol{P}\}+\{\{\delta \varphi\}\} \cdot \llbracket \boldsymbol{P} \rrbracket$, Eq. (54) takes the form

$$
\begin{aligned}
& \int_{\mathcal{B}_{0}} \boldsymbol{P}: \operatorname{Grad} \delta \boldsymbol{\varphi} \mathrm{d} V-\int_{\partial \mathcal{B}_{0}^{\mathrm{N}}} \delta \boldsymbol{\varphi} \cdot \widehat{\boldsymbol{b}}_{\mathrm{N}}^{\mathrm{p}} \mathrm{d} A \\
& +\int_{\mathcal{I}_{0}} \llbracket \delta \varphi \rrbracket \cdot \underbrace{\{\{\boldsymbol{P}\} \cdot \overline{\boldsymbol{N}}}_{\boldsymbol{T}}+\underbrace{[\{\delta \varphi\}\}-\delta \bar{\varphi}]}_{=\mathbf{0}} \cdot \llbracket \boldsymbol{P} \rrbracket \cdot \overline{\boldsymbol{N}} \mathrm{d} A \\
& -\int_{\mathcal{B}_{0}} \delta \boldsymbol{\varphi} \cdot \boldsymbol{b}^{\mathrm{p}} \mathrm{d} V+\int_{\mathcal{I}_{0}} \overline{\boldsymbol{P}}: \overline{\operatorname{Grad}} \delta \overline{\boldsymbol{\varphi}} \mathrm{d} A \\
& -\int_{\partial \mathcal{I}_{0}^{\mathrm{N}}} \delta \overline{\boldsymbol{\varphi}} \cdot \widetilde{\boldsymbol{b}}_{\mathrm{N}}^{\mathrm{p}} \mathrm{d} L-\int_{\mathcal{I}_{0}} \delta \overline{\boldsymbol{\varphi}} \cdot \overline{\boldsymbol{b}}^{\mathrm{p}} \mathrm{d} A=0 .
\end{aligned}
$$

Since $\llbracket \delta \varphi \rrbracket \neq \mathbf{0}$, for non-coherent interfaces, and $\{\{\delta \varphi\}=$ $\delta \overline{\boldsymbol{\varphi}}$, Eq. (55) simplifies to the weak form Eq. (32).

\section{References}

1. Alfano G, Crisfield MA (2001) Finite element interface models for the delamination analysis of laminated composites: mechanical and computational issues. Int J Numer Meth Eng 50:1701-1736

2. Alfano G, Sacco E (2006) Combining interface damage and friction in a cohesive-zone model. Int J Numer Meth Eng 68(5):542-582

3. Allix O, Corigliano A (1996) Modeling and simulation of crack propagation in mixed-modes interlaminar fracture specimens. Int $\mathrm{J}$ Fract 77(2):111-140

4. Allix O, Corigliano A (1999) Geometrical and interfacial nonlinearities in the analysis of delamination in composites. Int J Solids Struct 36(15):2189-2216

5. Allix O, Ladevéze P, Corigliano A (1995) Damage analysis of interlaminar fracture specimens. Compos. Struct. 31(1):61-74

6. Barenblatt GI (1962) The mathematical theory of equilibrium cracks in brittle fracture. Adv Appl Mech 7(1):55-129

7. Benveniste Y (2006) A general interface model for a threedimensional curved thin anisotropic interphase between two anisotropic media. J Mech Phys Solids 54(4):708-734

8. Benveniste Y (2013) Models of thin interphases and the effective medium approximation in composite media with curvilinearly anisotropic coated inclusions. Int J Eng Sci 72:140-154

9. Benveniste Y, Miloh T (2001) Imperfect soft and stiff interfaces in two-dimensional elasticity. Mech Mater 33(6):309-323

10. Bolzon G, Corigliano A (1997) A discrete formulation for elastic solids with damaging interfaces. Comput Methods Appl Mech Eng 140(3-4):329-359

11. Camacho GT, Ortiz M (1996) Computational modelling of impact damage in brittle materials. Int J Solids Struct 33(2):2899-2938

12. Cammarata RC (1997) Surface and interface stress effects on interfacial and nanostructured materials. Mater Sci Eng A 237(2):180184

13. Catlin A, Walker WP (1960) Mechanical properties of thin singlecrystal gold films. J Appl Phys 31(12):2135-2139
14. Cazes F, Coret M, Combescure A, Gravouil A (2009) A thermodynamic method for the construction of a cohesive law from a nonlocal damage model. Int J Solids Struct 46(6):1476-1490

15. Cervenka J, Saouma VE (1995) Discrete crack modeling in concrete structures. In: Wittmann FH (eds) Fracture mechanics of concrete structures, proceedings FRAMCOS-2

16. Chaboche JL, Girard R, Schaff A (1997) Numerical analysis of composite systems by using interphase/interface models. Comput Mech 20:3-11

17. Chatzigeorgiou G, Javili A, Steinmann P (2013) Multiscale modelling for composites with energetic interfaces at the micro- or nanoscale. Math Mech Solids 20(9):1130-1145

18. Chen J, Crisfield M, Kinloch AJ, Busso EP, Matthews FL, Qiu Y (1999) Predicting progressive delamination of composite material specimens via interface elements. Mech Compos Mater Struct 6(4):301-317

19. Corigliano A (1993) Formulation, identification and use of interface models in the numerical analysis of composite delamination. Int J Solids Struct 30(20):2779-2811

20. Daher N, Maugin GA (1986) The method of virtual power in continuum mechanics application to media presenting singular surfaces and interfaces. Acta Mech 60(3-4):217-240

21. Davydov D, Javili A, Steinmann P (2013) On molecular statics and surface-enhanced continuum modeling of nano-structures. Comput Mater Sci 69:510-519

22. dell'Isola F, Romano A (1987) On the derivation of thermomechanical balance equations for continuous systems with a nonmaterial interface. Int J Eng Sci 25:1459-1468

23. Dingreville R, Qu J, Cherkaoui M (2005) Surface free energy and its effect on the elastic behavior of nano-sized particles, wires and films. J Mech Phys Solids 53(8):1827-1854

24. Duan HL, Karihaloo BL (2007) Effective thermal conductivities of heterogeneous media containing multiple imperfectly bonded inclusions. Phys Rev B 75(6):064206

25. Duan HL, Wang J, Huang ZP, Karihaloo BL (2005a) Eshelby formalism for nano-inhomogeneities. Proc R Soc A 461(2062):33353353

26. Duan HL, Wang J, Huang ZP, Karihaloo BL (2005b) Sizedependent effective elastic constants of solids containing nanoinhomogeneities with interface stress. J Mech Phys Solids 53(7):1574-1596

27. Duan HL, Wang J, Karihaloo BL (2009) Theory of elasticity at the nanoscale. Adv Appl Mech 42:1-68

28. Dugdale DS (1960) Yielding of steel sheets containing slits. J Mech Phys Solids 8(2):100-104

29. Esmaeili A, Javili A, Steinmann P (2016a) Highly-conductive energetic coherent interfaces subject to in-plane degradation. Math Mech Solids. doi:10.1177/1081286516642818

30. Esmaeili A, Javili A, Steinmann P (2016b) A thermo-mechanical cohesive zone model accounting for mechanically energetic Kapitza interfaces. Int J Solids Struct 92-93:29-44

31. Fagerström M, Larsson R (2005) Theory and numerics for finite deformation fracture modelling using strong discontinuities. Int $\mathrm{J}$ Numer Meth Eng 66:911-948

32. Fagerström M, Larsson R (2008) A thermo-mechanical cohesive zone formulation for ductile fracture. J Mech Phys Solids 56(10):3037-3058

33. Fartash A, Fullerton EE, Schuller IK, Bobbin SE, Wagner JW, Cammarata RC, Kumar S, Grimsditch M (1991) Evidence for the supermodulus effect and enhanced hardness in metallic superlattices. Phys Rev B 44:13760-13763

34. Fischer FD, Svoboda J (2010) Stresses in hollow nanoparticles. Int J Solids Struct 47(20):2799-2805

35. Fleischhauer R, Behnke R, Kaliske M (2013) A thermomechanical interface element formulation for finite deformations. Comput Mech 52(5):1039-1058 
36. Fried E, Todres R (2005) Mind the gap: the shape of the free surface of a rubber-like material in proximity to a rigid contactor. J Elast 80(1-3):97-151

37. Gasser TC, Holzapfel GA (2003) Geometrically non-linear and consistently linearized embedded strong discontinuity models for 3D problems with an application to the dissection analysis of soft biological tissues. Comput Methods Appl Mech Eng 192(4748):5059-5098

38. Gurtin ME, Murdoch AI (1975) A continuum theory of elastic material surfaces. Arch Ration Mech Anal 57(4):291-323

39. Gurtin ME, Weissmüller J, Larché F (1998) A general theory of curved deformable interfaces in solids at equilibrium. Philos Mag A 78(5):1093-1109

40. Hillerborg A, Modéer M, Petersson P-E (1976) Analysis of crack formation and crack growth in concrete by means of fracture mechanics and finite elements. Cem Concr Res 6(6):773-781

41. Huang ZP, Sun L (2007) Size-dependent effective properties of a heterogeneous material with interface energy effect: from finite deformation theory to infinitesimal strain analysis. Acta Mech 190(1-4):151-163

42. Ijaz H, Asad M, Gornet L, Alam SY (2014) Prediction of delamination crack growth in carbon/fiber epoxy composite laminates using non-local interface damage model. Mech Ind 15(4):293-300

43. Javili A, Steinmann P (2009) A finite element framework for continua with boundary energies. Part I: the two-dimensional case. Comput Methods Appl Mech Eng 198(27-29):2198-2208

44. Javili A, Steinmann P (2010a) A finite element framework for continua with boundary energies. Part II: the three-dimensional case. Comput Methods Appl Mech Eng 199(9-12):755-765

45. Javili A, Steinmann P (2010b) On thermomechanical solids with boundary structures. Int J Solids Struct 47(24):3245-3253

46. Javili A, McBride A, Steinmann P (2012) Numerical modelling of thermomechanical solids with mechanically energetic (generalised) Kapitza interfaces. Comput Mater Sci 65:542-551

47. Javili A, dell' Isola F, Steinmann P (2013a) Geometrically nonlinear higher-gradient elasticity with energetic boundaries. J Mech Phys Solids 61(12):2381-2401

48. Javili A, McBride A, Steinmann P (2013b) Thermomechanics of solids with lower-dimensional energetics: on the importance of surface, interface, and curve structures at the nanoscale. A unifying review. Appl Mech Rev 65(1):010802

49. Javili A, McBride A, Steinmann P (2013c) Numerical modelling of thermomechanical solids with highly conductive energetic interfaces. Int J Numer Meth Eng 93(5):551-574

50. Javili A, Kaessmair S, Steinmann P (2014a) General imperfect interfaces. Comput Methods Appl Mech Eng 275:76-97

51. Javili A, McBride A, Steinmann P, Reddy BD (2014b) A unified computational framework for bulk and surface elasticity theory: a curvilinear-coordinate-based finite element methodology. Comput Mech 54(3):745-762

52. Jirásek M, Belytschko T (2002) Computational resolution of strong discontinuities. In: Proceedings of fifth world congress on computational mechanics, WCCM V, Vienna University of Technology, Austria

53. Ladevèze P, Allix O, Gornet L, Lévêque D, Perret L (1998) A computational damage mechanics approach for laminates: identification and comparison with experimental results. Stud Appl Mech 46:481-500

54. Levitas VI, Javanbakht M (2010) Surface tension and energy in multivariant martensitic transformations: phase-field theory, simulations, and model of coherent interface. Phys Rev Lett 105(16): 165701

55. Lin G, Geubelle PH, Sottos NR (2001) Simulation of fiber debonding with friction in a model composite pushout test. Int J Solids Struct 38(46-47):8547-8562
56. Mergheim J, Steinmann P (2006) A geometrically nonlinear FE approach for the simulation of strong and weak discontinuities. Comput Methods Appl Mech Eng 195:5037-5052

57. Mi Y, Crisfield MA, Davies GAO, Hellweg HB (1998) Progressive delamination using interface elements. J Compos Mater 32(14):1246-1272

58. Moeckel GP (1975) Thermodynamics of an interface. Arch Ration Mech Anal 57(3):255-280

59. Mosler J, Scheider I (2011) A thermodynamically and variationally consistent class of damage-type cohesive models. J Mech Phys Solids 59(8):1647-1668

60. Mosler J, Stanković L, Radulović R (2011) Efficient modeling of localized material failure by means of a variationally consistent embedded strong discontinuity approach. Int J Numer Meth Eng 88(10):1008-1041

61. Murdoch AI (1976) A thermodynamical theory of elastic material interfaces. Q J Mech Appl Math 29(3):245-275

62. Needleman A (1987) A continuum model for void nucleation by inclusion debonding. J Appl Mech 54(3):525-531

63. Nguyen O, Repetto EA, Ortiz M, Radovitzky RA (2001) A cohesive model of fatigue crack growth. Int J Fract 110:351-369

64. Ortiz M, Pandolfi A (1999) Finite-deformation irreversible cohesive elements for three-dimensional crack-propagation analysis. Int J Numer Meth Eng 44:1267-1282

65. Ottosen NS (1986) Thermodynamic consequences of strain softening in tension. J Eng Mech 112(11):1152-1164

66. Ottosen NS, Ristinmaa M (2013) Thermodynamically based fictitious crack/interface model for general normal and shear loading. Int J Solids Struct 50(22-23):3555-3561

67. Ottosen NS, Ristinmaa M, Mosler J (2015) Fundamental physical principles and cohesive zone models at finite displacementslimitations and possibilities. Int J Solids Struct 53:70-79

68. Ottosen NS, Ristinmaa M, Mosler J (2016) Framework for noncoherent interface models at finite displacement jumps and finite strains. J Mech Phys Solids 90:124-141 ISSN 0022-5096

69. Özdemir I, Brekelmans WAM, Geers MGD (2010) A Thermomechanical cohesive zone model. Comput Mech 46:735-745

70. Pandolfi A, Krysl P, Ortiz M (1999) Finite element simulation of ring expansion and fragmentation: the capturing of length and time scales through cohesive models of fracture. Int J Fract 95(14):279-297

71. Park HS, Klein PA (2007) Surface Cauchy-Born analysis of surface stress effects on metallic nanowires. Phys Rev B 75:085408

72. Park K, Paulino GH (2011) Cohesive zone models: a critical review of traction-separation relationships across fracture surfaces. Appl Mech Rev 64(6):060802

73. Park K, Paulino GH, Roesler JR (2009) A unified potential-based cohesive model of mixed-mode fracture. J Mech Phys Solids 57(6):891-908

74. Parrinello F, Failla B, Borino G (2009) Cohesive-frictional interface constitutive model. Int J Solids Struct 46(13):2680-2692

75. Raous M (2011) Interface models coupling adhesion and friction. C R Mec 339(7-8):491-501

76. Roe KL, Siegmund T (2003) An irreversible cohesive zone model for interface fatigue crack growth simulation. Eng Fract Mech 70:209-232

77. Schellekens JCJ, Borst RD (1993) A non-linear finite element approach for the analysis of mode-I free edge delamination in composites. Int J Solids Struct 30(9):1239-1253

78. Sharma P, Ganti S (2004) Size-dependent Eshelbys tensor for embedded nano-inclusions incorporating surface/interface energies. J Appl Mech 71(5):663-671

79. Sharma P, Wheeler LT (2007) Size-dependent elastic state of ellipsoidal nano-inclusions incorporating surface/interface tension. J Appl Mech 74(3):447-454 
80. Sharma P, Ganti S, Bhate N (2003) Effect of surfaces on the sizedependent elastic state of nano-inhomogeneities. Appl Phys Lett 82(4):535-537

81. Simo JC, Hughes TJR (1998) Computational inelasticity. Springer, New York

82. Steigmann DJ, Ogden RW (1999) Elastic surface-substrate interactions. Proc R Soc Lond A 455(1982):437-474

83. Steinmann $P$ (1999) Formulation and computation of geometrically non-linear gradient damage. Int J Numer Meth Eng 46(5):757-779

84. Steinmann P (2008) On boundary potential energies in deformational and configurational mechanics. J Mech Phys Solids 56(3):772-800

85. Steinmann P, Larsson R, Runesson K (1997) On the localization properties of multiplicative hyperelasto-plastic continua with strong discontinuities. Int J Solids Struct 34(8):969-990

86. Tvergaard V (1990) Effect of fibre debonding in a whiskerreinforced metal. Mater Sci Eng A 125(2):203-213

87. Tvergaard V, Hutchinson JW (1992) The relation between crack growth resistance and fracture process parameters in elastic-plastic solids. J Mech Phys Solids 40(6):1377-1397

88. van den Bosch MJ, Schreurs PJG, Geers MGD (2006) An improved description of the exponential $\mathrm{Xu}$ and Needleman cohesive zone law for mixed-mode decohesion. Eng Fract Mech 73:1220-1234
89. van den Bosch MJ, Schreurs PJG, Geers MGD (2007) A cohesive zone model with a large displacement formulation accounting for interfacial fibrilation. Eur J Mech A Solids 26:1-19

90. van den Bosch MJ, Schreurs PJG, Geers MGD (2008) Identification and characterization of delamination in polymer coated metal sheet. J Mech Phys Solids 56(11):3259-3276

91. Willam K, Rhee I, Shing B (2004) Interface damage model for thermomechanical degradation of heterogeneous materials. Comput Methods Appl Mech Eng 193(30):3327-3350

92. Xu X-P, Needleman A (1993) Void nucleation by inclusion debonding in a crystal matrix. Modell Simul Mater Sci Eng 1(2):111

93. Xu X-P, Needleman A (1994) Numerical simulations of fast crack growth in brittle solids. J Mech Plys Solid 42(9):1397-1434

94. Yvonnet J, Mitrushchenkov A, Chambaud G, He Q-C (2011) Finite element model of ionic nanowires with size-dependent mechanical properties determined by ab initio calculations. Comput Methods Appl Mech Eng 200(5-8):614-625 